\title{
Wightman function and the Casimir effect for a Robin sphere in a constant curvature space
}

\author{
S. Bellucci ${ }^{1, a}$, A. A. Saharian ${ }^{2, b}$, N. A. Saharyan ${ }^{2}$ \\ ${ }^{1}$ INFN, Laboratori Nazionali di Frascati, Via Enrico Fermi 40, 00044 Frascati, Italy \\ 2 Department of Physics, Yerevan State University, 1 Alex Manoogian Street, 0025 Yerevan, Armenia
}

Received: 1 August 2014 / Accepted: 27 August 2014 / Published online: 11 September 2014

(C) The Author(s) 2014. This article is published with open access at Springerlink.com

\begin{abstract}
We evaluate the Wightman function, the mean field squared and the vacuum expectation value of the energy-momentum tensor for a scalar field with the Robin boundary condition on a spherical shell in the background of a constant negative curvature space. For the coefficient in the boundary condition there is a critical value above which the scalar vacuum becomes unstable. In both the interior and the exterior regions, the vacuum expectation values are decomposed into the boundary-free and sphere-induced contributions. For the latter, rapidly convergent integral representations are provided. In the region inside the sphere, the eigenvalues are expressed in terms of the zeros of the combination of the associated Legendre function and its derivative and the decomposition is achieved by making use of the Abel-Plana type summation formula for the series over these zeros. The sphere-induced contribution to the vacuum expectation value of the field squared is negative for the Dirichlet boundary condition and positive for the Neumann one. At distances from the sphere larger than the curvature scale of the background space the suppression of the vacuum fluctuations in the gravitational field corresponding to the negative curvature space is stronger compared with the case of the Minkowskian bulk. In particular, the decay of the vacuum expectation values with the distance is exponential for both massive and massless fields. The corresponding results are generalized for spaces with spherical bubbles and for cosmological models with negative curvature spaces.
\end{abstract}

\section{Introduction}

In the absence of a reliable theory for quantum gravity, the influence of the gravitational field on quantum matter is investigated within the framework of semiclassical theory.

\footnotetext{
a e-mail: bellucci@lnf.infn.it

b e-mail: saharian@ysu.am
}

In the latter, the gravitational field is considered as the classical background and the back-reaction of quantum effects is described by quasiclassical Einstein equations with the expectation value of the energy-momentum tensor for quantum fields in the right-hand side (for reviews see [1-5]). Though the quantum field theory in curved spacetime is not a fundamental theory, it should have a very broad range of physical applicability, from the smallest distance accessible in experiments to cosmological scales. This hybrid but very useful scheme is an important intermediate step to the development of quantum gravity. Among the most interesting effects in this field are the particle production and the vacuum polarization by strong gravitational fields. A wellknown example is the thermal radiation emitted by black holes.

The investigation of quantum effects in curved backgrounds is motivated by several reasons. During the cosmological expansion of the Universe, the back-reaction of the particles created by a time dependent gravitational field at early stages leads to a rapid isotropization of the expansion. According to the inflationary scenario, the quantum vacuum fluctuations of a scalar field, amplified by the gravitational field during the quasi-de Sitter like expansion, serve as seeds for the large scale structure formation in the Universe $[6,7]$. The information on the properties of these fluctuations are encoded in the thermal anisotropies of the cosmic microwave background, which are measured with high accuracy by a number of recent cosmological projects. An important feature of quantum field theory in curved backgrounds is a possible breakdown of the energy conditions in the formulations of Hawking-Penrose singularity theorems, by the expectation value of the energy-momentum tensor of quantum fields. This opens a possibility to solve the singularity problem within the framework of classical general relativity. An additional motivation for the study of quantum fieldtheoretical effects in curved backgrounds appeared recently in condensed matter physics related to various analogous 
models. In particular, the long-wavelength properties of the electronic subsystem in a graphene sheet are well described by the Dirac-like model with the speed of light replaced by the Fermi velocity. In curved graphene structures (for example, in fullerenes) the corresponding field theory is formulated on curved geometry and the curvature effects should be taken into account in the calculations of physical properties of these structures $[8,9]$.

A large number of problems, in addition to the curvature of the background spacetime, involve boundaries on which the operator of a quantum field obeys the prescribed boundary conditions. The physical nature of the boundaries can be different. The examples are macroscopic bodies in QED, edges of graphene sheets in nanoribbons, interfaces separating different phases of a physical system, horizons in gravitational physics, the branes in higher-dimensional fieldtheoretical models. Another motivation for studying boundaries comes from the quantization of closed cosmological models (see, for instance [10]) and from the Euclidean quantum gravity [11]. Among the most interesting macroscopic manifestations of the influence of boundaries on the properties of a quantum field is the Casimir effect (see Refs. [12-16]). It arises as a consequence of the modification of the quantum fluctuations spectrum by the boundary conditions imposed on the field. As a result of this, the expectation values of physical observables are changed and forces arise acting on the constraining boundaries. The effect for scalar, fermionic, and vector fields is investigated in various bulk and boundary geometries and for various types of boundary conditions.

An interesting topic in the investigations of the Casimir effect is the dependence of the physical characteristics of the vacuum, like the energy density and stresses, on the geometry of the background spacetime. The evaluation of these characteristics requires the knowledge of a complete set of modes for a quantum field and exact results can be provided for highly symmetric bulk and boundary geometries only. In particular, motivated by the problems of the radion stabilization and the cosmological constant generation in braneworld models, the Casimir effect for planar boundaries in antide Sitter space has been widely discussed [17-31]. Higherdimensional generalizations of the anti-de Sitter background having compact internal spaces have been considered as well [32-39]. Another class of exactly solvable Casimir problems corresponds to de Sitter spacetime. Various geometries of boundaries in this background have been considered [4049]. In inflationary coordinates the corresponding metric is time-dependent and, in general, in addition to the diagonal component, the vacuum energy-momentum tensor has a nonzero off-diagonal component that describes an energy flux along the direction normal to the boundary. The Casimir effect for the electromagnetic field in the geometry of parallel conducting plates in Friedmann-Robertson-Walker cos- mologies with flat spatial sections and with power-law scale factors is discussed in [50].

In the present paper we consider the change in the properties of the scalar vacuum induced by a spherical shell with the Robin boundary condition on the background of a negatively curved constant curvature space. Historically, the investigation of the Casimir effect with a spherical boundary was motivated by the semiclassical model of an electron [51], where the vacuum quantum fluctuations of the electromagnetic field are responsible for Poincaré stress stabilizing the charged particle. Currently the configurations involving spherical boundaries are among the most popular ones in both the theoretical and experimental investigations of the Casimir effect (see [12-16] and references therein). Here, we are interested in combined effects of the gravitational field and spherical boundary on the local characteristics of the scalar vacuum. We shall consider a free field theory and in this case all the properties of the vacuum state are obtained from two-point functions. Our choice of the positive-frequency Wightman function is motivated by the fact that this function will also determine the response of a Unruh-De Witt detector (see, for instance, [1]). As local characteristics of the vacuum state we shall investigate the vacuum expectation values of the field squared and the energy-momentum tensor. The latter serves as a source in the right-hand side of semiclassical Einstein equations and plays an important role in considerations of the back-reaction from quantum effects on the background geometry.

The paper is organized as follows. In Sect. 2, we describe the bulk and boundary geometries. The positive-frequency Wightman function is evaluated for the boundary-free space, inside and outside a spherical boundary. By making use of this function, in Sect. 3, the sphere-induced contribution in the vacuum expectation value of the field squared is evaluated and its properties are investigated in asymptotic regions of the parameters. The expectation value of the energy-momentum tensor is studied in Sect. 4 for the both interior and exterior regions. In Sect. 5, we consider the background spacetime with the geometry described by distinct metric tensors inside and outside a spherical boundary. Two cases are investigated. In the first one the interior geometry is described by a general spherically symmetric static metric and the exterior metric corresponds to a constant negative curvature space. An example is considered with an interior Minkowskian geometry. In the second case, a constant curvature space is realized in the interior region whereas the exterior geometry is the Minkowski one. The main results are summarized in Sect. 6 . For the interior geometry with a negative constant curvature space, the eigenmodes of a quantum scalar field with the Robin boundary condition are expressed in terms of the zeros of the associated Legendre function with respect to its order. In Appendix A, we discuss the properties of these zeros. The expression of the Wightman function inside the 
spherical shell contains the summation over these zeros. In Appendix B, by making use of the generalized Abel-Plana formula, a summation formula is derived for the series of this type.

\section{Wightman function}

\subsection{Geometry of the problem}

We consider a quantum scalar field ${ }^{1} \varphi(x)$ with the curvature coupling parameter $\xi$. The most important special cases of this parameter $\xi=0$ and $\xi=\xi_{D}=(D-1) /(4 D)$ correspond to minimally and conformally coupled scalars, respectively. The field equation has the form

$\left(\nabla_{l} \nabla^{l}+m^{2}+\xi \mathcal{R}\right) \varphi(x)=0$,

where $\mathcal{R}$ is the scalar curvature for the background spacetime. The background geometry is described by the line element

$\mathrm{d} s^{2}=\mathrm{d} t^{2}-a^{2}\left(\mathrm{~d} r^{2}+\sinh ^{2} r \mathrm{~d} \Omega_{D-1}^{2}\right)$,

with a constant $a$ and with $d \Omega_{D-1}^{2}$ being the line element on the $(D-1)$-dimensional sphere, $S^{D-1}$. The corresponding angular coordinates we denote by $(\vartheta, \phi)$ with $\vartheta=\left(\theta_{1}, \ldots, \theta_{n}\right), n=D-2$, and $0 \leqslant \theta_{k} \leqslant \pi, k=1, \ldots, n$, $0 \leqslant \phi \leqslant 2 \pi$. The spatial part of the line element (2.2) describes a constant negative curvature space. The spaces with negative curvature play a significant role in cosmology and in holographic theories. For the Ricci scalar corresponding to (2.2) one has $\mathcal{R}=-D(D-1) / a^{2}$. Note that in Eq. (2.1) the curvature coupling term appears in the form of the effective mass squared $m^{2}-D(D-1) \xi / a^{2}$. Depending on the value of the curvature coupling parameter, the latter can be either negative or positive.

Quantum effects on the background of constant curvature spaces have been widely discussed in the literature (see, for instance, $[1,2,12-16,53,54])$. These effects play an important role in the physics of the early Universe, in inflationary models, and in a number of condensed matter systems described by effective curved geometries. Here we are interested in combined effects of the background gravitational field and boundaries on the properties of the quantum vacuum for a scalar field. As a boundary geometry we consider a spherical shell on which the field operator obeys the Robin boundary condition

$\left(A+B n^{l} \nabla_{l}\right) \varphi(x)=0, r=r_{0}$,

${ }^{1}$ In quantum field theory the fields are operator-valued distributions [52]. where $r_{0}$ is the sphere radius, $A$ and $B$ are constants, and $n^{l}$ is the unit inward normal to the sphere, $n^{l}=-\delta_{(j)} \delta_{1}^{l} / a$, $j=i, e$, with $\delta_{(i)}=1$ for the interior region and $\delta_{(e)}=-1$ for the exterior region. With this, for $B \neq 0$, the boundary condition can also be written in the form $\left(\beta-\delta_{(j)} \partial_{r}\right) \varphi(x)=$ $0, r=r_{0}$, with the notation

$\beta=a A / B$

Of course, all the physical results will depend on this ratio. We wrote the condition in the form (2.3) to keep the transition to the special cases of the Dirichlet $(B=0)$ and the Neumann ( $A=0$ ) boundary conditions transparent.

For a free field theory all the properties of the quantum vacuum are contained in two-point functions. Here we shall consider the positive-frequency Wightman function defined as the vacuum expectation value $W\left(x, x^{\prime}\right)=$ $\left\langle 0\left|\varphi(x) \varphi\left(x^{\prime}\right)\right| 0\right\rangle$, where $|0\rangle$ stands for the vacuum state. The expectation values of physical characteristics bilinear in the field operator, such as the field squared and the energymomentum tensor, are obtained from this function in the coincidence limit. In addition to this, the positive-frequency Wightman function determines the response function for the Unruh-De Witt particle detector in a given state of motion [1].

Let $\left\{\varphi_{\alpha}(x), \varphi_{\alpha}^{*}(x)\right\}$ be a complete set of normalized positive- and negative-energy mode functions obeying the field equation (2.1) and the boundary condition (2.3). Here, the collective index $\alpha$ is the set of quantum numbers specifying the solutions. For the problem under consideration, the positive-energy mode functions can be presented in the factorized form

$\varphi_{\alpha}(x)=R_{l}(r) Y\left(m_{k} ; \vartheta, \phi\right) \mathrm{e}^{-i E t}$,

where $Y\left(m_{k} ; \vartheta, \phi\right)$ is the spherical harmonic of degree $l$. For the angular quantum numbers one has $l=0,1,2 \ldots$, $m_{k}=\left(m_{0}=l, m_{1}, \ldots, m_{n}\right)$, where $m_{1}, m_{2}, \ldots, m_{n}$ are integers obeying the relations

$0 \leqslant m_{n-1} \leqslant m_{n-2} \leqslant \cdots \leqslant m_{1} \leqslant l$,

and $-m_{n-1} \leqslant m_{n} \leqslant m_{n-1}$. By taking into account the equation for the spherical harmonics,

$\Delta_{(\vartheta, \phi)} Y\left(m_{k} ; \vartheta, \phi\right)=-b_{l} Y\left(m_{k} ; \vartheta, \phi\right)$,

with

$b_{l}=l(l+D-2)$,

from the field equation (2.1) for the radial function $R_{l}(r)$ one gets 


$$
\begin{aligned}
& \frac{1}{\sinh ^{D-1} r} \frac{\mathrm{d}}{\mathrm{d} r}\left(\sinh ^{D-1} r \frac{\mathrm{d} R_{l}}{\mathrm{~d} r}\right) \\
& +\left[\left(E^{2}-m^{2}\right) a^{2}+D(D-1) \xi-\frac{b_{l}}{\sinh ^{2} r}\right] R_{l}(r)=0 .
\end{aligned}
$$

Introducing a new function $f_{l}(r)=\sinh ^{D / 2-1}(r) R_{l}(r)$, we can see that the general solution for this function is a linear combination of the associated Legendre functions of the first and second kinds, $P_{i z-1 / 2}^{-\mu}(\cosh r)$ and $Q_{i z-1 / 2}^{-\mu}(\cosh r)$ (here the definition of the associated Legendre functions follows that of Ref. [55]) with the order and degree determined by

$\mu=l+D / 2-1, z^{2}=E^{2} a^{2}-z_{m}^{2}$.

Here and in what follows we use the notation

$z_{m}=\sqrt{m^{2} a^{2}-D(D-1)\left(\xi-\xi_{D}\right)}$.

The relative coefficient in the linear combination depends on the spatial region under consideration and will be determined below. Now, as a set of quantum numbers $\alpha$, specifying the mode functions in Eq. (2.5), we can take $\alpha=$ $\left(z, l, m_{1}, \ldots, m_{n}\right)$. The energy is expressed in terms of $z$ by the formula

$E(z)=a^{-1} \sqrt{z^{2}+z_{m}^{2}}$

Below we shall assume that $z_{m}^{2} \geqslant 0$. In particular, this condition is satisfied in the most important special cases of minimally and conformally coupled fields. If it is not obeyed there are modes with imaginary values of the energy, which signal the vacuum instability. Note that the condition $z_{m}^{2} \geqslant 0$ is different from the non-negativity condition for the effective mass squared $m^{2}-D(D-1) \xi / a^{2}$.

The modes (2.5) are normalized by the standard orthonormalization condition

$\int \mathrm{d}^{D} x \sqrt{|g|} \varphi_{\alpha}(x) \varphi_{\alpha^{\prime}}^{*}(x)=\frac{\delta_{\alpha \alpha^{\prime}}}{2 E}$,

where the symbol $\delta_{\alpha \alpha^{\prime}}$ is understood as the Kronecker delta for discrete indices and as the Dirac delta function for continuous ones. With this normalization, the Wightman function is evaluated by using the mode-sum,

$W\left(x, x^{\prime}\right)=\sum_{\alpha} \varphi_{\alpha}(x) \varphi_{\alpha}^{*}\left(x^{\prime}\right)$,

where $\sum_{\alpha}$ stands for the summation over discrete quantum numbers and for the integration over continuous ones. In what follows, the Wightman function will be decomposed into the boundary-free and sphere-induced contributions. For that reason we first consider the boundary-free geometry.
2.2 Wightman function in the boundary-free geometry

For the boundary-free geometry the solution of the radial equation, regular at the origin, is given in terms of the associated Legendre function of the first kind. The corresponding positive-energy mode functions have the form

$\varphi_{\alpha}^{(0)}(x)=A_{\alpha}^{(0)} p_{i z-1 / 2}^{-\mu}(u) Y\left(m_{k} ; \vartheta, \phi\right) \mathrm{e}^{-i E t}$,

with $0 \leqslant z<\infty$ and with the notations

$p_{v}^{-\mu}(u)=\frac{P_{v}^{-\mu}(u)}{\left(u^{2}-1\right)^{(D-2) / 4}}, u=\cosh r$.

From the property $P_{i z-1 / 2}^{-\mu}(u)=P_{-i z-1 / 2}^{-\mu}(u)$ it follows that the radial function in $(2.15)$ is real.

In the case $z=z^{\prime}$ the normalization integral (2.13), with the integration over $r \in[0, \infty)$, diverges and, hence, the main contribution comes from large values $r$. By using the asymptotic formula for the associated Legendre function for large values of the argument, we see that

$$
\begin{aligned}
& \int_{1}^{\infty} \mathrm{d} u P_{i z-1 / 2}^{-\mu}(u) P_{i z^{\prime}-1 / 2}^{-\mu}(u) \\
& \quad=\frac{\pi}{z \sinh (\pi z)} \frac{\delta\left(z-z^{\prime}\right)}{|\Gamma(\mu+1 / 2+i z)|^{2}} .
\end{aligned}
$$

With this result, for the normalization coefficient in (2.15) one finds

$\left|A_{\alpha}^{(0)}\right|^{2}=\frac{z \sinh (\pi z)}{2 \pi N\left(m_{k}\right) a^{D} E}|\Gamma(\mu+1 / 2+i z)|^{2}$.

Here we have used the result $\int d \Omega\left|Y\left(m_{k} ; \vartheta, \phi\right)\right|^{2}=N\left(m_{k}\right)$ (the specific form for $N\left(m_{k}\right)$ is given in Ref. [56] and will not be required in the following discussion). For $D=3$, the modes (2.15) with the coefficient (2.18) reduce to the ones discussed in Refs. [2,57].

Substituting the functions (2.15) into the mode-sum (2.14), we use the addition theorem

$\sum_{m_{k}} \frac{Y\left(m_{k} ; \vartheta, \phi\right)}{N\left(m_{k}\right)} Y^{*}\left(m_{k} ; \vartheta^{\prime}, \phi^{\prime}\right)=\frac{(2 l+n)}{n S_{D}} C_{l}^{n / 2}(\cos \theta)$,

where $S_{D}=2 \pi^{D / 2} / \Gamma(D / 2)$ is the surface area of the unit sphere in $D$-dimensional space, $C_{l}^{n / 2}(\cos \theta)$ is the Gegenbauer polynomial and $\theta$ is the angle between the directions determined by $(\vartheta, \phi)$ and $\left(\vartheta^{\prime}, \phi^{\prime}\right)$. For the corresponding Wightman function we find the formula

$W_{0}\left(x, x^{\prime}\right)=\frac{a^{-D}}{2 \pi n S_{D}} \sum_{l=0}^{\infty}(2 l+n) C_{l}^{n / 2}(\cos \theta)$ 


$$
\begin{aligned}
& \times \int_{0}^{\infty} \mathrm{d} z z \sinh (\pi z) \\
& \times|\Gamma(i z+\mu+1 / 2)|^{2} p_{i z-1 / 2}^{-\mu}(u) p_{i z-1 / 2}^{-\mu}\left(u^{\prime}\right) \frac{\mathrm{e}^{-i E(z) \Delta t}}{E(z)},
\end{aligned}
$$

with $u^{\prime}=\cosh r^{\prime}$ and with $E(z)$ given by Eq. (2.12).

\subsection{Wightman function inside the sphere}

In the presence of a spherical shell with the radius $r_{0}$, the mode functions for the interior region, $r<r_{0}$, are written in the form similar to Eq. (2.15):

$\varphi_{\alpha}(x)=A_{\alpha} p_{i z-1 / 2}^{-\mu}(u) Y\left(m_{k} ; \vartheta, \phi\right) \mathrm{e}^{-i E(z) t}$.

Imposing the boundary condition (2.3), we see that the eigenvalues for the quantum number $z$ are solutions of the equation

$\bar{P}_{i z-1 / 2}^{-\mu}\left(u_{0}\right)=0, u_{0}=\cosh r_{0}$.

Here and in the discussion below, for a given function $F(u)$ we use the notation

$\bar{F}(u)=A(u) F(u)+B(u) F^{\prime}(u)$,

with the coefficients

$$
\begin{gathered}
A(u)=A \sqrt{u^{2}-1}+(D / 2-1) \delta_{(j)} \frac{B}{a} u, \\
B(u)=-\delta_{(j)} \frac{B}{a}\left(u^{2}-1\right) .
\end{gathered}
$$

By using the recurrence relation for the associated Legendre function, one has an equivalent expression,

$$
\begin{aligned}
\bar{P}_{i z-1 / 2}^{-\mu}(u)= & \left(A \sqrt{u^{2}-1}-\delta_{(j)} l \frac{B}{a} u\right) P_{i z-1 / 2}^{-\mu}(u) \\
& +\delta_{(j)} \frac{B}{a}\left[(1 / 2+\mu)^{2}+z^{2}\right] \sqrt{u^{2}-1} P_{i z-1 / 2}^{-\mu-1}(u) .
\end{aligned}
$$

For given $r_{0}$ and $l$, Eq. (2.22) has an infinite set of positive roots with respect to $z$. We shall denote them, arranged in ascending order of magnitude, as $z=z_{k}, k=1,2, \ldots$. Note that these roots do not depend on the curvature coupling parameter and on the mass of the field. As is discussed in Appendix A, in addition to the real roots with respect to $z$, depending on the value of $\beta$, a purely imaginary root may appear. First we consider the case when all the roots are real. This case is realized for $\beta<\beta_{0}^{(1)}\left(u_{0}\right)$ (see Appendix A).

Substituting the mode functions (2.21) into the orthonormalization condition, with the integration over the region inside the spherical shell, for the normalization coefficient one finds

$$
\left|A_{\alpha}\right|^{-2}=2 E a^{D} N\left(m_{k}\right) \int_{1}^{u_{0}} \mathrm{~d} u\left|P_{i z-1 / 2}^{-\mu}(u)\right|^{2} .
$$

The integral is evaluated by using the integration formula

$$
\begin{aligned}
\int_{1}^{b} \mathrm{~d} u\left[P_{i z-1 / 2}^{-\mu}(u)\right]^{2}= & \frac{b^{2}-1}{2 z}\left\{\left[\partial_{z} P_{i z-1 / 2}^{-\mu}(b)\right] \partial_{b} P_{i z-1 / 2}^{-\mu}(b)\right. \\
& \left.-P_{i z-1 / 2}^{-\mu}(b) \partial_{z} \partial_{b} P_{i z-1 / 2}^{-\mu}(b)\right\} .
\end{aligned}
$$

For the roots of Eq. (2.22), $z=z_{k}$, and for $b=u_{0}$, the expression in the figure braces is equal to $-P_{i z-1 / 2}^{-\mu}\left(u_{0}\right) \partial_{z} \bar{P}_{i z-1 / 2}^{-\mu}\left(u_{0}\right) / B\left(u_{0}\right)$. With this result, the normalization coefficient is written in terms of $T_{\mu}(z, u)$, defined by Eq. (8.7) in Appendix B, by the expression

$$
\begin{aligned}
\left|A_{\alpha}\right|^{2}= & \frac{\mathrm{e}^{i \mu \pi} z T_{\mu}\left(z, u_{0}\right)}{\pi a^{D} N\left(m_{k}\right) E(z)} \\
& \times \Gamma(\mu+i z+1 / 2) \Gamma(\mu-i z+1 / 2),
\end{aligned}
$$

with $z=z_{k}$. Having determined the normalized mode functions, we turn to the evaluation of the Wightman function in the region inside the sphere with the help of Eq. (2.14). Substituting the eigenfunctions, one finds

$$
\begin{aligned}
& W\left(x, x^{\prime}\right)=\frac{a^{-D}}{\pi n S_{D}} \sum_{l=0}^{\infty}(2 l+n) C_{l}^{n / 2}(\cos \theta) \mathrm{e}^{i \mu \pi} \\
& \times \sum_{k=1}^{\infty} z_{k} T_{\mu}\left(z_{k}, u_{0}\right) \\
& \times\left|\Gamma\left(i z_{k}+\mu+1 / 2\right)\right|^{2} p_{i z_{k}-1 / 2}^{-\mu}(u) p_{i z_{k}-1 / 2}^{-\mu}\left(u^{\prime}\right) \frac{\mathrm{e}^{-i E\left(z_{k}\right) \Delta t}}{E\left(z_{k}\right)},
\end{aligned}
$$

where $u^{\prime}=\sinh r^{\prime}$ and $\Delta t=t-t^{\prime}$. The roots $z_{k}$ are given implicitly and the representation (2.29) is not convenient for the evaluation of the vacuum expectation values of the field squared and the energy-momentum tensor. Additionally, the terms with large $k$ are highly oscillatory. Both these difficulties are avoided by applying to the series over $k$ the summation formula (8.4) with the function

$$
\begin{aligned}
h(z)= & z \Gamma(\mu+i z+1 / 2) \Gamma(\mu-i z+1 / 2) P_{i z-1 / 2}^{-\mu}(u) \\
& \times P_{i z-1 / 2}^{-\mu}\left(u^{\prime}\right) \frac{\mathrm{e}^{-i E(z) \Delta t}}{E(z)} .
\end{aligned}
$$

The corresponding conditions are obeyed if $r+r^{\prime}+\Delta t / a<$ $2 r_{0}$. Note that the function (2.30) has branch points $z=$ $\pm i z_{m}$.

The part in the Wightman function obtained from the first integral in the right-hand side of Eq. (8.4) coincides with the boundary-free function $W_{0}\left(x, x^{\prime}\right)$. In the second integral, the part over the interval $\left(0, z_{m}\right)$ vanishes and for the Wightman function we find 


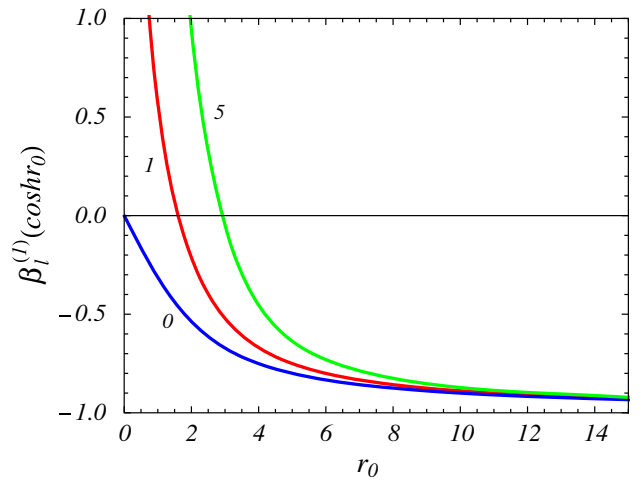

Fig. 1 The critical values of the Robin coefficient for the appearance of the purely imaginary roots of the eigenvalue equation (2.22) in the interior region (left panel) and for the appearance of the bound states

$W\left(x, x^{\prime}\right)=W_{0}\left(x, x^{\prime}\right)+W_{\mathrm{s}}\left(x, x^{\prime}\right)$,

where for the sphere-induced part one has

$$
\begin{gathered}
W_{\mathrm{s}}\left(x, x^{\prime}\right)=-\frac{a^{1-D}}{\pi n S_{D}} \sum_{l=0}^{\infty}(2 l+n) C_{l}^{n / 2}(\cos \theta) \mathrm{e}^{-i \mu \pi} \int_{z_{m}}^{\infty} \mathrm{d} z z \\
\times \frac{\bar{Q}_{z-1 / 2}^{\mu}\left(u_{0}\right)}{\bar{P}_{z-1 / 2}^{-\mu}\left(u_{0}\right)} p_{z-1 / 2}^{-\mu}(u) p_{z-1 / 2}^{-\mu}\left(u^{\prime}\right) \frac{\cosh \left(\sqrt{z^{2}-z_{m}^{2}} \Delta t / a\right)}{\sqrt{z^{2}-z_{m}^{2}}} .
\end{gathered}
$$

In deriving this formula we have used the relation [55]

$Q_{z-1 / 2}^{-\mu}(u)=\mathrm{e}^{-2 i \mu \pi} \frac{\Gamma(z-\mu+1 / 2)}{\Gamma(z+\mu+1 / 2)} Q_{x-1 / 2}^{\mu}(u)$,

for the associated Legendre function. Equation (2.32) provides our final expression for the sphere-induced part of the Wightman function in the interior region. In this form knowledge of the roots $z_{k}$ is not required and for $r+r^{\prime}+\Delta t / a<2 r_{0}$ the integrand exponentially decays in the upper limit. In the special case $D=3$ and for the Dirichlet boundary condition, by taking into account Eq. (2.33), we see that Eq. (2.32) is reduced to the expression given in Ref. [58].

We have considered the case when all the zeros $z_{k}$ of the function $\bar{P}_{i z-1 / 2}^{-\mu}\left(u_{0}\right)$ are real. As is noticed in Appendix A, for given $r_{0}$ and $l$, starting from some critical value of $\beta$, for $\beta>\beta_{l}^{(1)}\left(\cosh r_{0}\right)$, a single purely imaginary zero $z=i \eta_{l}$, $\eta_{l}>0$, appears. This zero first appears for the angular mode $l=0$ and, hence, for a given $r_{0}$ the purely imaginary zeros are absent if $\beta<\beta_{0}^{(1)}\left(\cosh r_{0}\right)$. In order to have a stable vacuum state we assume that $E\left(i \eta_{l}\right)>0$ or $\eta_{l}<z_{m}$. In the left panel of Fig. 1, for the spatial dimension $D=3$, we have plotted the critical value $\beta_{l}^{(1)}\left(\cosh r_{0}\right)$ for the Robin coefficient, at which the purely imaginary zero appears, as a function of the sphere radius $r_{0}$ for $l=0,1,5$ (the numbers near the curves).

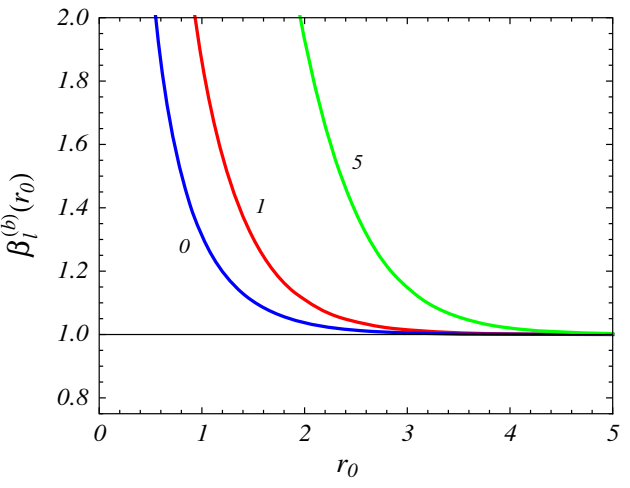

in the exterior region (right panel) versus the radius of the sphere. The graphs are plotted for the spatial dimension $D=3$

In the presence of the imaginary eigenmode, the corresponding contribution in the Wightman function should be added to the expression (2.29). This contribution is evaluated in a way similar to the one we have used for the modes with real $z$ and has the form

$$
\begin{aligned}
W^{(\mathrm{im})}\left(x, x^{\prime}\right)= & \sum_{l=0}^{\infty} \frac{(2 l+n)}{n S_{D} a^{D}} \frac{C_{l}^{n / 2}(\cos \theta)}{u_{0}^{2}-1} \frac{\eta_{l} B\left(u_{0}\right)}{E\left(i \eta_{l}\right)} \\
& \times \frac{p_{\eta_{l}-1 / 2}^{-\mu}(u) p_{\eta_{l}-1 / 2}^{-\mu}\left(u^{\prime}\right) \mathrm{e}^{-i E\left(i \eta_{l}\right) \Delta t}}{\left.P_{\eta_{l}-1 / 2}^{-\mu}\left(u_{0}\right) \partial_{\eta} \bar{P}_{\eta-1 / 2}^{-\mu}\left(u_{0}\right)\right|_{\eta=\eta_{l}}} .
\end{aligned}
$$

In the part of the Wightman function corresponding to the contribution of the modes with real $z$, given by Eq. (2.29), again we apply the summation formula (8.4). But now, as is explained in Appendix A, in the right-hand side of this formula we should add the term (8.10). By taking into account the expression (2.30) for the function $h(z)$, we can see that this term exactly cancels the contribution (2.34). Hence, the expression for the Wightman function given by Eq. (2.32) is also valid in the presence of the purely imaginary zeros for the function $\bar{P}_{i z-1 / 2}^{-\mu}\left(u_{0}\right)$ with $\eta_{l}<z_{m}$.

As an additional check of Eq. (2.32) let us consider the Minkowskian limit, which corresponds to large values of $a$ with fixed $a r=\rho$, where $\rho$ is the Minkowskian radial coordinate. Firstly we introduce in Eq. (2.32) a new integration variable $y=z / a$ and then use the asymptotic formulas

$$
\begin{aligned}
P_{v}^{-\mu}[\cosh (x / v)] & \approx \frac{I_{\mu}(x)}{v^{\mu}}, \\
Q_{\nu}^{\mu}[\cosh (x / v)] & \approx \mathrm{e}^{i \mu \pi} v^{\mu} K_{\mu}(x),
\end{aligned}
$$

valid for $v \gg 1$. By taking also into account that for large $a$ one has $z_{m}=a m$, we can see that from Eq. (2.32) the expression for the Wightman function is obtained inside a Robin sphere in Minkowski spacetime with the radius $\rho_{0}=$ $a r_{0}$ (see Ref. [59]). 


\subsection{Exterior region}

In the region outside the sphere, $r>r_{0}$, the radial part of the mode functions is a linear combination of the two linear independent solutions of the Legendre equation. As such solutions it is convenient to take the functions $Q_{i z-1 / 2}^{-\mu}(\cosh r)$ and $Q_{-i z-1 / 2}^{-\mu}(\cosh r)$. The relative coefficient of the linear combination of these functions is determined from the boundary condition (2.3) and the mode functions are written in the form

$\varphi_{\alpha}(x)=B_{\alpha} \frac{Z_{i z}^{-\mu}(u) Y\left(m_{k} ; \vartheta, \phi\right)}{\left(u^{2}-1\right)^{(D-2) / 4}} \mathrm{e}^{-i E(z) t}$,

where, as before, $u=\cosh r$, and

$$
\begin{aligned}
Z_{i z}^{-\mu}(u)= & \bar{Q}_{i z-1 / 2}^{-\mu}\left(u_{0}\right) Q_{-i z-1 / 2}^{-\mu}(u) \\
& -\bar{Q}_{-i z-1 / 2}^{-\mu}\left(u_{0}\right) Q_{i z-1 / 2}^{-\mu}(u) .
\end{aligned}
$$

The notation with the bar is defined by Eq. (2.23) with the coefficients given in Eq. (2.24). In addition to the modes with real $z$, depending on the values of the coefficients in the Robin boundary condition, the modes can be present with purely imaginary $z$. These modes correspond to bound states. First we consider the case when the bound states are absent.

From the normalization condition (2.13), for the modes (2.36) we have

$B_{\alpha}^{2} \int_{u_{0}}^{\infty} \mathrm{d} u Z_{i z}^{-\mu}(u)\left[Z_{i z^{\prime}}^{-\mu}(u)\right]^{*}=\frac{\delta\left(z-z^{\prime}\right)}{2 a^{D} N\left(m_{k}\right) E(z)}$.

The $u$-integral diverges in the upper limit for $z=z^{\prime}$ and, hence, the main contribution comes from large values $u$. So, we can replace the associated Legendre functions with the arguments $u$ by their asymptotic expressions for large values of the argument. To find this asymptotic we use the expression of the associated Legendre function in terms of the hypergeometric function,

$$
\begin{aligned}
& P_{i z-1 / 2}^{-\mu}(\cosh r)=\sqrt{\pi} \frac{\Gamma(1 / 2+i z-\mu)}{\mathrm{e}^{i \mu \pi} \Gamma(1+i z)} \frac{\mathrm{e}^{-(i z+1 / 2) r}}{\left(1-\mathrm{e}^{-2 r}\right)^{\mu}} \\
& \quad \times F\left(1 / 2-\mu, 1 / 2+i z-\mu ; 1+i z ; \mathrm{e}^{-2 r}\right) .
\end{aligned}
$$

From this, for large values $u$ to the leading order we find

$$
\begin{aligned}
Q_{i z-1 / 2}^{-\mu}(u) \approx & \sqrt{\pi} \frac{\Gamma(1 / 2+i z-\mu)}{\mathrm{e}^{i \mu \pi} \Gamma(1+i z)} \frac{\mathrm{e}^{-i z \ln (2 u)}}{\sqrt{2 u}} \\
& \times\left(1+O\left(1 / u^{2}\right)\right) .
\end{aligned}
$$

Substituting this asymptotic into the integral (2.38), for the normalization coefficient one finds

$B_{\alpha}^{2}=\frac{V^{-2}(z)}{2 \pi^{2} a^{D} N\left(m_{k}\right) E}$, where

$V(z)=\left|\mathrm{e}^{i \mu \pi} \bar{Q}_{i z-1 / 2}^{-\mu}\left(u_{0}\right) \frac{\Gamma(1 / 2-i z-\mu)}{\Gamma(1-i z)}\right|$.

By using the mode functions (2.36) with the normalization coefficient (2.41), from the mode-sum formula (2.14) for the Wightman function in the exterior region we find

$$
\begin{aligned}
W\left(x, x^{\prime}\right)= & \frac{a^{-D}}{2 \pi^{2} n S_{D}} \sum_{l=0}^{\infty} \frac{(2 l+n) C_{l}^{n / 2}(\cos \theta)}{\left(\sinh r \sinh r^{\prime}\right)^{D / 2-1}} \\
& \times \int_{0}^{\infty} \mathrm{d} z \frac{\mathrm{e}^{-i E \Delta t}}{E(z) V^{2}(z)} Z_{i z}^{-\mu}(u)\left[Z_{i z}^{-\mu}\left(u^{\prime}\right)\right]^{*}
\end{aligned}
$$

Introducing instead of the function $Q_{-i z-1 / 2}^{-\mu}(u)$ the function $P_{i z-1 / 2}^{-\mu}(u)$ and using the properties of the gamma function, the Wightman function is also expressed in the form

$W\left(x, x^{\prime}\right)=\frac{a^{-D}}{2 \pi n S_{D}} \sum_{l=0}^{\infty} \frac{(2 l+n) C_{l}^{n / 2}(\cos \theta)}{\left(\sinh r \sinh r^{\prime}\right)^{D / 2-1}}$

$\times \int_{0}^{\infty} \mathrm{d} z z \sinh (\pi z)$

$\times|\Gamma(i z+\mu+1 / 2)|^{2} \frac{Y_{i z-1 / 2}^{-\mu}(u)\left[Y_{i z-1 / 2}^{-\mu}\left(u^{\prime}\right)\right]^{*}}{\bar{Q}_{i z-1 / 2}^{-\mu}\left(u_{0}\right)\left[\bar{Q}_{i z-1 / 2}^{-\mu}\left(u_{0}\right)\right]^{*}} \frac{\mathrm{e}^{-i E(z) \Delta t}}{E(z)}$,

where

$Y_{v}^{-\mu}(u)=P_{v}^{-\mu}(u) \bar{Q}_{v}^{-\mu}\left(u_{0}\right)-\bar{P}_{v}^{-\mu}\left(u_{0}\right) Q_{v}^{-\mu}(u)$.

Here we are interested in the part of the Wightman function induced by the spherical shell. To obtain this contribution we subtract from the function (2.44) the Wightman function for the geometry without boundaries, which is given by Eq. (2.20). For the further evaluation of the difference we use the identity

$$
\begin{aligned}
& \frac{Y_{i z-1 / 2}^{-\mu}(u)\left[Y_{i z-1 / 2}^{-\mu}\left(u^{\prime}\right)\right]^{*}}{\bar{Q}_{i z-1 / 2}^{-\mu}\left(u_{0}\right)\left[\bar{Q}_{i z-1 / 2}^{-\mu}\left(u_{0}\right)\right]^{*}}-P_{i z-1 / 2}^{-\mu}(u) P_{i z-1 / 2}^{-\mu}\left(u^{\prime}\right)=\frac{\mathrm{e}^{i \mu \pi}}{\pi i \sinh (\pi z)} \\
& \quad \times \sum_{s= \pm 1} s \cos [\pi(s i z-\mu)] \frac{\bar{P}_{i z}^{-\mu}-1 / 2}{\bar{Q}_{s i z-1 / 2}^{-\mu}\left(u_{0}\right)} Q_{s i z-1 / 2}^{-\mu}(u) Q_{s i z-1 / 2}^{-\mu}\left(u^{\prime}\right) .
\end{aligned}
$$

Substituting this in the expression for the part of the Wightman function induced by the sphere, we rotate the integration contour over $z$ by the angle $\pi / 2$ for the term with $s=-1$ and by the angle $-\pi / 2$ for the term with $s=1$. This leads to the following result: 


$$
\begin{gathered}
W_{\mathrm{s}}\left(x, x^{\prime}\right)=-\frac{a^{1-D}}{\pi n S_{D}} \sum_{l=0}^{\infty}(2 l+n) C_{l}^{n / 2}(\cos \theta) \mathrm{e}^{-i \mu \pi} \int_{z_{m}}^{\infty} \mathrm{d} z z \\
\times \frac{\bar{P}_{z-1 / 2}^{-\mu}\left(u_{0}\right)}{\bar{Q}_{z-1 / 2}^{\mu}\left(u_{0}\right)} q_{z-1 / 2}^{\mu}(u) q_{z-1 / 2}^{\mu}\left(u^{\prime}\right) \frac{\cosh \left(\Delta t \sqrt{z^{2}-z_{m}^{2}}\right)}{\sqrt{z^{2}-z_{m}^{2}}},
\end{gathered}
$$

with the notation

$q_{\nu}^{\mu}(u)=\frac{Q_{\nu}^{\mu}(u)}{\left(u^{2}-1\right)^{(D-2) / 4}}$.

Here, once again, we have used Eq. (2.33). Comparing the expression (2.47) with Eq. (2.32), we see that the sphereinduced parts in the Wightman function for interior and exterior regions are obtained from each other by the replacements $P_{z-1 / 2}^{-\mu} \rightleftarrows Q_{z-1 / 2}^{\mu}$.

Now, let us turn to the case when bound states are present. For these states $z=i \eta, \eta>0$, and the mode functions are given by the expression

$\varphi_{\alpha}(x)=B_{\mathrm{b} \alpha} q_{\eta-1 / 2}^{-\mu}(u) Y\left(m_{k} ; \vartheta, \phi\right) \mathrm{e}^{-i E(i \eta) t}$,

with the energy $E(i \eta)=a^{-1} \sqrt{z_{m}^{2}-\eta^{2}}$. In order to have a stable vacuum state we assume that $\eta<z_{m}$. From the boundary condition (2.3) we see that, for a given $r_{0}$, the possible bound states are solutions of the equation

$\bar{Q}_{\eta-1 / 2}^{-\mu}\left(u_{0}\right)=0$.

Note that this equation does not involve the field mass and the curvature coupling parameter. The numerical analysis shows that there are no bound states for $\beta \leqslant(D-1) / 2$. With fixed $r_{0}$ and $l$, a single bound state appears started from some critical value $\beta_{l}^{(b)}\left(r_{0}\right)>(D-1) / 2$. We shall denote the corresponding root by $\eta=\eta_{(b) l}$. This critical value increases with increasing $l$. In the right panel of Fig. 1 , for the $D=3$ case, we have plotted $\beta_{l}^{(b)}\left(r_{0}\right)$ as a function of the sphere radius $r_{0}$ for several values of $l$ (numbers near the curves). From the asymptotic expression for the function $Q_{\eta-1 / 2}^{-\mu}\left(u_{0}\right)$ it can be seen that $\beta_{l}^{(b)}\left(r_{0}\right) \rightarrow(D-1) / 2$ for $r_{0} \rightarrow \infty$.

In order to find the normalization coefficient in Eq. (2.49) we use the integration formula

$$
\begin{aligned}
& \int_{u}^{\infty} \mathrm{d} x\left[Q_{\eta-1 / 2}^{-\mu}(x)\right]^{2}=\frac{u^{2}-1}{2 \eta} \\
& \times\left\{\left[\partial_{\eta} Q_{\eta-1 / 2}^{-\mu}(u)\right] \partial_{u} Q_{\eta-1 / 2}^{-\mu}(u)-Q_{\eta-1 / 2}^{-\mu}(u) \partial_{\eta} \partial_{u} Q_{\eta-1 / 2}^{-\mu}(u)\right\} .
\end{aligned}
$$

This formula is obtained by making use of the differential equation for the associated Legendre function. For the roots of Eq. (2.50) from Eq. (2.51) we get

$$
\begin{aligned}
& \int_{u_{0}}^{\infty} \mathrm{d} x\left[Q_{\eta-1 / 2}^{-\mu}(x)\right]^{2} \\
& \quad=\left.\frac{1-u_{0}^{2}}{2 \eta B\left(u_{0}\right)} Q_{\eta-1 / 2}^{-\mu}\left(u_{0}\right) \partial_{\eta} \bar{Q}_{\eta-1 / 2}^{-\mu}\left(u_{0}\right)\right|_{\eta=\eta_{(b) l}} .
\end{aligned}
$$

By using this result, the normalization coefficient for the bound states is presented in the form

$$
\left|B_{\mathrm{b} \alpha}\right|^{2}=\left.\frac{a^{-D} \eta B\left(u_{0}\right)}{N\left(m_{k}\right) E(\eta)} \frac{\left(1-u_{0}^{2}\right)^{-1}}{Q_{\eta-1 / 2}^{-\mu}\left(u_{0}\right) \partial_{\eta} \bar{Q}_{\eta-1 / 2}^{-\mu}\left(u_{0}\right)}\right|_{\eta=\eta_{(b) l}} .
$$

With this coefficient, for the contribution of the bound states to the Wightman function one gets

$$
\begin{gathered}
W^{(\mathrm{bs})}\left(x, x^{\prime}\right)=\sum_{l=0}^{\infty} \frac{(2 l+n)}{n S_{D} a^{D}} \frac{B\left(u_{0}\right) C_{l}^{n / 2}(\cos \theta)}{E(\eta)\left(1-u_{0}^{2}\right)} \\
\times\left.\frac{\eta q_{\eta-1 / 2}^{-\mu}(u) q_{\eta-1 / 2}^{-\mu}\left(u^{\prime}\right)}{Q_{\eta-1 / 2}^{-\mu}\left(u_{0}\right) \partial_{\eta} \bar{Q}_{\eta-1 / 2}^{-\mu}\left(u_{0}\right)} \mathrm{e}^{-i E(i \eta) \Delta t}\right|_{\eta=\eta_{(b) l}} .
\end{gathered}
$$

In the presence of the bound states, the contribution to the Wightman function from the modes with real $z$ is still given by Eq. (2.44). Again, for this expression we use the identity (2.46). The difference from the previous case arises at the step when one rotates the integration contour over $z$. Now, the integrands have simple poles on the imaginary axis corresponding to the zeros of the functions $\bar{Q}_{s i z-1 / 2}^{-\mu}\left(u_{0}\right)$ in the denominator of Eq. (2.46). Rotating the integration contours for the terms with $s=-1$ and $s=1$, we escape these poles by semicircles of a small radius in the right-half plane. The integrals over these semicircles combine in the residue at the point $z=\mathrm{e}^{\pi i / 2} \eta_{(b) l}$. As a result, for the part in the Wightman function coming from the modes with real $z$ we get the expression (2.47) plus the contribution coming from the residue at the pole $z=\mathrm{e}^{\pi i / 2} \eta_{(b) l}$. Now, it can be seen that the latter is exactly canceled by the contribution from the bound state, given by Eq. (2.54). Hence, we conclude that the expression (2.47) for the shell-induced Wightman function in the exterior region is valid in the presence of bound states as well.

Similarly to the case of the interior region, we can see that in the limit $a \rightarrow \infty$ from (2.47) the corresponding expression is obtained for the sphere in Minkowski spacetime.

\section{Vacuum expectation value of the field squared}

Among the most important local characteristics of the vacuum state are the vacuum expectation values of the field squared and the energy-momentum tensor. We start with the mean field squared. It is obtained from the Wightman function in the coincidence limit of the arguments. This limit is divergent and a renormalization procedure is required. In 
curved backgrounds the structure of divergences is determined by the local geometry. In the problem under consideration, for points away from the sphere, the local geometry is the same as in the boundary-free case and, hence, the divergences in the local physical characteristics are the same as well. From here it follows that the renormalization procedure for these characteristics is the same as that in the boundaryfree geometry. In the expressions given above for the both interior and exterior regions we have explicitly decomposed the Wightman function into the boundary-free and sphereinduced parts. For points outside the sphere, the renormalization is needed for the boundary-free vacuum expectation values only and the sphere-induced parts are directly obtained from the corresponding Wigthman function in the coincidence limit.

For the renormalized mean field squared we get

$$
\left\langle\varphi^{2}\right\rangle=\left\langle\varphi^{2}\right\rangle_{0}+\left\langle\varphi^{2}\right\rangle_{\mathrm{s}}
$$

where $\left\langle\varphi^{2}\right\rangle_{0}$ is the renormalized vacuum expectation value in the boundary-free space and the part $\left\langle\varphi^{2}\right\rangle_{\mathrm{S}}$ is induced by the sphere. For the latter, by taking into account that $C_{l}^{n / 2}(1)=$ $\Gamma(l+n) /[\Gamma(n) l !]$, in the interior region from Eq. (2.32) one finds

$$
\begin{aligned}
\left\langle\varphi^{2}\right\rangle_{\mathrm{s}}= & -\frac{a^{1-D}}{\pi S_{D}} \sum_{l=0}^{\infty} D_{l} \mathrm{e}^{-i \mu \pi} \\
& \times \int_{z m}^{\infty} \mathrm{d} z z \frac{\bar{Q}_{z-1 / 2}^{\mu}\left(u_{0}\right)}{\bar{P}_{z-1 / 2}^{-\mu}\left(u_{0}\right)} \frac{\left[p_{z-1 / 2}^{-\mu}(u)\right]^{2}}{\sqrt{z^{2}-z_{m}^{2}}},
\end{aligned}
$$

where

$D_{l}=2 \mu \frac{\Gamma(l+D-2)}{\Gamma(D-1) l !}$,

is the degeneracy of the angular mode with given $l$. For the functions in the integrand, we have numerically checked that $\mathrm{e}^{-i \mu \pi} \bar{Q}_{z-1 / 2}^{\mu}\left(u_{0}\right)>0$ in both special cases of the Dirichlet and the Neumann boundary conditions, whereas $\bar{P}_{z-1 / 2}^{-\mu}\left(u_{0}\right)>0$ for the Dirichlet boundary condition and $\bar{P}_{z-1 / 2}^{-\mu}\left(u_{0}\right)<0$ for the Neumann one. Consequently, the sphere-induced vacuum expectation value of the field squared is negative for the Dirichlet boundary condition and positive for the Neumann boundary condition.

By using the asymptotic formulas

$$
\begin{aligned}
P_{z-1 / 2}^{-\mu}(u) & \approx \frac{z^{-\mu-1 / 2} \mathrm{e}^{r z}}{\sqrt{2 \pi \sinh r}}(1+O(1 / z)), \\
Q_{z-1 / 2}^{\mu}(u) & \approx \mathrm{e}^{i \mu \pi} \frac{\pi z^{\mu-1 / 2} \mathrm{e}^{-r z}}{\sqrt{2 \pi \sinh r}}(1+O(1 / z)),
\end{aligned}
$$

valid for $z \gg 1$, we see that the integrand behaves as $\mathrm{e}^{2 z\left(r-r_{0}\right)} / z$. Hence, the sphere-induced vacuum expectation value (3.2) diverges on the boundary. In order to find the leading term in the asymptotic expansion over the distance from the sphere, we note that for points close to the boundary the dominant contribution in Eq. (3.2) comes from large values of $z$ and $l$. In this case, instead of Eq. (3.4), we need to use the uniform asymptotic expansions for the associated Legendre functions for large values of both $z$ and $\mu$. The latter can be obtained from Eq. (2.35) by making use of the uniform asymptotic expansions for the modified Bessel functions (see, for instance, Ref. [55]). In this way, to the leading order, we get

$$
\left\langle\varphi^{2}\right\rangle_{\mathrm{s}} \approx-\frac{a^{1-D} \Gamma((D-1) / 2) \delta_{B}}{(4 \pi)^{(D+1) / 2}\left(r_{0}-r\right)^{D-1}}\left(1+O\left(r_{0}-r\right)\right),
$$

with $\delta_{B}=2 \delta_{0 B}-1$. As is seen, near the sphere the boundaryinduced vacuum expectation value has opposite signs for Dirichlet and non-Dirichlet boundary conditions. By taking into account that $a\left(r_{0}-r\right)$ is the proper distance from the sphere, we see that the leading term coincides with that for the sphere in Minkowski bulk. Of course, this is natural, because near the sphere the contribution of the modes with the wavelengths smaller than the curvature radius dominate and they are relatively insensitive to the background geometry.

At the sphere center, by taking into account the asymptotic $p_{z-1 / 2}^{-\mu}(u) \approx 2^{-\mu} r^{l} / \Gamma(\mu+1)$ for $r \rightarrow 0$, we see that the $l=0$ mode contributes only:

$$
\begin{aligned}
& \left.\left\langle\varphi^{2}\right\rangle_{\mathrm{s}}\right|_{r=0}=\frac{(2 a)^{1-D} \mathrm{e}^{-i \pi D / 2}}{\pi^{D / 2+1} \Gamma(D / 2)} \\
& \times \int_{z_{m}}^{\infty} \mathrm{d} z \frac{z}{\sqrt{z^{2}-z_{m}^{2}}} \frac{\bar{Q}_{z-1 / 2}^{D / 2-1}\left(u_{0}\right)}{\bar{P}_{z-1 / 2}^{1-D / 2}\left(u_{0}\right)} .
\end{aligned}
$$

Near the center the contribution of the modes with higher $l$ decays as $r^{2 l}$. Note that Eq. (3.6) is further simplified for $D=3$. By taking into account the formulas

$$
\begin{gathered}
P_{z-1 / 2}^{-1 / 2}(u)=\frac{2 \sinh (z r)}{z \sqrt{2 \pi \sinh r}}, \\
Q_{z-1 / 2}^{1 / 2}(u)=\frac{i \pi \mathrm{e}^{-z r}}{\sqrt{2 \pi \sinh r}},
\end{gathered}
$$

we obtain

$$
\frac{\bar{Q}_{z-1 / 2}^{1 / 2}\left(u_{0}\right)}{\bar{P}_{z-1 / 2}^{-1 / 2}\left(u_{0}\right)}=\frac{i \pi z}{\frac{\beta+u_{0}-z}{\beta+u_{0}+z} \mathrm{e}^{2 z r_{0}}-1}
$$

Hence, for $D=3$, Eq. (3.6) is reduced to

$$
\left.\left\langle\varphi^{2}\right\rangle_{\mathrm{s}}\right|_{r=0}=-\frac{a^{-2}}{2 \pi^{2}} \int_{z_{m}}^{\infty} \mathrm{d} z \frac{z^{2}}{\sqrt{z^{2}-z_{m}^{2}}} \frac{1}{\frac{\beta+u_{0}-z}{\beta+u_{0}+z} \mathrm{e}^{2 z r_{0}}-1}
$$

with $z_{m}^{2}=m^{2} a^{2}+1-6 \xi$. 
In the discussion below we shall also need the covariant Dalambertian of the boundary-induced part:

$$
\begin{aligned}
& \nabla_{p} \nabla^{p}\left\langle\varphi^{2}\right\rangle_{\mathrm{s}}=\frac{2 a^{-1-D}}{\pi S_{D}} \sum_{l=0}^{\infty} D_{l} \mathrm{e}^{-i \mu \pi} \\
& \times \int_{z_{m}}^{\infty} \mathrm{d} z z \frac{\bar{Q}_{z-1 / 2}^{\mu}\left(u_{0}\right)}{\bar{P}_{z-1 / 2}^{-\mu}\left(u_{0}\right)} \frac{F\left[p_{z-1 / 2}^{-\mu}(u)\right]}{\sqrt{z^{2}-z_{m}^{2}}} .
\end{aligned}
$$

In this formula we have introduced the function

$$
\begin{aligned}
F[f(u)]= & \frac{1}{2}\left[\left(u^{2}-1\right) \partial_{u}^{2}+D u \partial_{u}\right] f^{2}(u) \\
= & \left(u^{2}-1\right) f^{\prime 2}(u) \\
& +\left[\frac{b_{l}}{u^{2}-1}-\frac{(D-1)^{2}}{4}+z^{2}\right] f^{2}(u) .
\end{aligned}
$$

In the second expression, in order to exclude the second order derivative, we have used the differential equation obeyed by the function $p_{z-1 / 2}^{-\mu}(u)$.

In the exterior region, the shell-induced contribution in the vacuum expectation value of the field squared is obtained from Eq. (2.47) in the coincidence limit:

$$
\begin{aligned}
\left\langle\varphi^{2}\right\rangle_{\mathrm{s}}= & -\frac{a^{1-D}}{\pi S_{D}} \sum_{l=0}^{\infty} D_{l} \mathrm{e}^{-i \mu \pi} \\
& \times \int_{z_{m}}^{\infty} \mathrm{d} z z \frac{\bar{P}_{z-1 / 2}^{-\mu}\left(u_{0}\right)}{\bar{Q}_{z-1 / 2}^{\mu}\left(u_{0}\right)} \frac{\left[q_{z-1 / 2}^{\mu}(u)\right]^{2}}{\sqrt{z^{2}-z_{m}^{2}}} .
\end{aligned}
$$

This quantity is negative for the Dirichlet boundary condition and positive for the Neumann boundary condition. For points near the sphere, the leading term in the asymptotic expansion of this expectation value is given by Eq. (3.5) with $r_{0}-r$ replaced by $r-r_{0}$. In this limit, the effects of the background gravitational field are small. The curvature effects are crucial at distances from the sphere larger than the curvature radius of the background space. This corresponds to the limit of large $r$ with a fixed value of the sphere radius $r_{0}$. For $r \gg 1$ we use the approximate formula

$q_{z-1 / 2}^{\mu}(u) \approx 2^{D / 2-1} \frac{\mathrm{e}^{i \mu \pi} \sqrt{\pi} \Gamma(z+1 / 2+\mu)}{\Gamma(z+1) \mathrm{e}^{(z+(D-1) / 2) r}}\left(1+O\left(1 / u^{2}\right)\right)$.

With this asymptotic, the dominant contribution in the integral of Eq. (3.12) comes from the region near the lower limit of the integration. For $z_{m}>0$, assuming that $z_{m} r \gg 1$, to the leading order we get

$$
\begin{aligned}
\left\langle\varphi^{2}\right\rangle_{\mathrm{s}} \approx & -\frac{2^{D-3} \sqrt{\pi z_{m} / r}}{S_{D} a^{D-1} \mathrm{e}^{\left(2 z_{m}+D-1\right) r}} \sum_{l=0}^{\infty} D_{l} \frac{\bar{P}_{z_{m}-1 / 2}^{-\mu}\left(u_{0}\right)}{\bar{Q}_{z_{m}-1 / 2}^{\mu}\left(u_{0}\right)} \\
& \times \mathrm{e}^{i \mu \pi} \frac{\Gamma^{2}\left(z_{m}+l+D / 2\right)}{\Gamma^{2}\left(z_{m}+1\right)}(1+O(1 / r)) .
\end{aligned}
$$

The boundary-induced vacuum expectation value is exponentially small and the suppression factor depends on the curvature coupling parameter. For $z_{m}=0$ the leading term in the asymptotic expansion at large distance takes the form

$$
\begin{aligned}
\left\langle\varphi^{2}\right\rangle_{\mathrm{s}} \approx & -\frac{2^{D-3} a^{1-D}}{S_{D} r \mathrm{e}^{(D-1) r}} \sum_{l=0}^{\infty} D_{l} \mathrm{e}^{i \mu \pi} \frac{\bar{P}_{-1 / 2}^{-\mu}\left(u_{0}\right)}{\bar{Q}_{-1 / 2}^{\mu}\left(u_{0}\right)} \\
& \times \Gamma^{2}(l+D / 2)(1+O(1 / r)) .
\end{aligned}
$$

In this case the decay is weaker, though again exponential. In particular, for both minimally and conformally coupled massless scalars the suppression of the boundary-induced vacuum expectation values at large distances is exponential.

For a spherical boundary in Minkowski bulk and for a massive field the vacuum expectation value at large distances is suppressed by the factor $\mathrm{e}^{-2 m \rho}$ with $\rho$ being the Minkowskian radial coordinate. In this case the suppression factor does not depend on the curvature coupling. For a massless field in Minkowski spacetime, the dominant contribution at large distances comes from the angular mode $l=0$ and the decay of the expectation value is of power-law, like $1 / \rho^{2 D-3}$ for $D \geqslant 3$. This shows that the suppression of the vacuum fluctuations in the gravitational field corresponding to the negative curvature space is stronger compared with the case of the Minkowskian bulk. A similar feature is observed in the geometry of planar boundaries on anti-de Sitter bulk (see Ref. [17-31]), then yielding another example of a negative curvature space. For de Sitter geometry, having a positive curvature, the situation is essentially different: the boundaryinduced contributions in the local vacuum expectation values decay at large distances as a power-law for both massive and massless fields.

\section{Energy-momentum tensor}

For the evaluation of the vacuum expectation value of the energy-momentum tensor we use the formula

$$
\begin{aligned}
& \left\langle T_{i k}\right\rangle=\lim _{x^{\prime} \rightarrow x} \partial_{i^{\prime}} \partial_{k} W\left(x, x^{\prime}\right) \\
& \quad+\left[(\xi-1 / 4) g_{i k} \nabla_{p} \nabla^{p}-\xi \nabla_{i} \nabla_{k}-\xi \mathcal{R}_{i k}\right]\left\langle\varphi^{2}\right\rangle,
\end{aligned}
$$

where $\mathcal{R}_{i k}$ is the Ricci tensor. For the geometry under consideration one has (no summation over $p$ ) $\mathcal{R}_{p}^{p}=-(D-1) / a^{2}$ for $p=1, \ldots, D$, and the remaining components vanish. In the right-hand side of Eq. (4.1) we have used the expression for the energy-momentum tensor for a scalar field, which differs from the standard one by the term which vanishes on the solutions of the field equation and does not contribute to the boundary-induced vacuum expectation value (see Ref. [60]).

By taking into account the expressions for the Wightman function and for the expectation value of the field squared 
from the previous section, the vacuum energy-momentum tensor is presented in the decomposed form

$$
\left\langle T_{i k}\right\rangle=\left\langle T_{i k}\right\rangle_{0}+\left\langle T_{i k}\right\rangle_{\mathrm{s}}
$$

where the first and second terms in the right-hand side correspond to the boundary-free and sphere-induced contributions. Again, for points away the sphere, the renormalization is needed for the first term only.

In the interior region, after straightforward calculations, we find that the vacuum expectation value of the energymomentum tensor is diagonal with the components (no summation over $k$ )

$$
\begin{aligned}
& \left\langle T_{k}^{k}\right\rangle_{\mathrm{s}}=\frac{a^{-1-D}}{\pi S_{D}} \sum_{l=0}^{\infty} D_{l} \mathrm{e}^{-i \mu \pi} \\
& \times \int_{z_{m}}^{\infty} \mathrm{d} z z \frac{\bar{Q}_{z-1 / 2}^{\mu}\left(u_{0}\right)}{\bar{P}_{z-1 / 2}^{-\mu}\left(u_{0}\right)} \frac{F^{(k)}\left[p_{z-1 / 2}^{-\mu}(u)\right]}{\sqrt{z^{2}-z_{m}^{2}}},
\end{aligned}
$$

where we have introduced the notations

$$
\begin{aligned}
& F^{(0)}[f(u)]=2(\xi-1 / 4) F[f(u)]+\left(z^{2}-z_{m}^{2}\right) f^{2}(u), \\
& F^{(1)}[f(u)]=\frac{1}{2} F[f(u)]+2(D-1) \xi u f(u) f^{\prime}(u) \\
& -\left[(D-1) \xi+\frac{b_{l}}{u^{2}-1}-\frac{(D-1)^{2}}{4}+z^{2}\right] f^{2}(u), \\
& F^{(k)}[f(u)]=2(\xi-1 / 4) F[f(u)]-2 \xi u f(u) f^{\prime}(u) \\
& +\frac{1}{D-1}\left[\frac{b_{l}}{u^{2}-1}-(D-1)^{2} \xi\right] f^{2}(u),
\end{aligned}
$$

with $F[f(u)]$ defined in Eq. (3.11) and $k=2,3, \ldots, D$ in the last expression.

We can check that the part in the vacuum expectation value of the energy-momentum tensor induced by the spherical shell satisfies the covariant conservation equation $\nabla_{k}\left\langle T_{i}^{k}\right\rangle_{\mathrm{s}}=$ 0 , which for the geometry under consideration takes the form

$\left(u^{2}-1\right) \frac{\partial}{\partial u}\left\langle T_{1}^{1}\right\rangle_{\mathrm{s}}+(D-1) u\left(\left\langle T_{1}^{1}\right\rangle_{\mathrm{s}}-\left\langle T_{2}^{2}\right\rangle_{\mathrm{s}}\right)=0$.

In addition, it can be seen that the boundary-induced parts in the vacuum expectation values satisfy the trace relation

$\left\langle T_{k}^{k}\right\rangle_{\mathrm{s}}=D\left(\xi-\xi_{D}\right) \nabla_{p} \nabla^{p}\left\langle\varphi^{2}\right\rangle_{\mathrm{s}}+m^{2}\left\langle\varphi^{2}\right\rangle_{\mathrm{s}}$

In particular, for a conformally coupled massless scalar field the boundary-induced part in the expectation value of the energy-momentum tensor is traceless. The trace anomalies are contained in the boundary-free part.

The sphere-induced contribution in the vacuum expectation value of the energy-momentum tensor diverges on the boundary. The leading terms in the expansion over the distance from the sphere for the energy density and parallel stresses are found in a way similar to that we have used for the field squared. They are given by (no summation over $k)$

$$
\left\langle T_{k}^{k}\right\rangle_{\mathrm{s}} \approx \frac{D \Gamma((D+1) / 2)\left(\xi-\xi_{D}\right)}{2^{D} \pi^{(D+1) / 2}\left[a\left(r_{0}-r\right)\right]^{D+1}} \delta_{B}\left(1+O\left(r_{0}-r\right)\right),
$$

for the components $k=0,2, \ldots$ Again, these leading terms coincide with those for a sphere in the Minkowski bulk. The leading term for the radial stress vanishes and the next to the leading terms in the relations (2.35) should be kept. Easier way is to use the continuity equation (4.5) with the result

$$
\left\langle T_{1}^{1}\right\rangle_{\mathrm{s}} \approx\left(1-\frac{1}{D}\right) \frac{u_{0}\left(r_{0}-r\right)}{\sqrt{u_{0}^{2}-1}}\left\langle T_{0}^{0}\right\rangle_{\mathrm{s}},
$$

with $\left\langle T_{0}^{0}\right\rangle_{\mathrm{s}}$ from Eq. (4.7).

At the sphere center only the terms with $l=0,1$ contribute, and one has (no summation over $k$ )

$$
\begin{aligned}
\left\langle T_{k}^{k}\right\rangle_{\mathrm{s}}= & \frac{a^{-1-D}}{\pi S_{D}} \int_{z_{m}}^{\infty} \mathrm{d} z \frac{z}{\sqrt{z^{2}-z_{m}^{2}}} \\
& \times \sum_{l=0}^{1} \frac{D_{l} \mathrm{e}^{-i \mu \pi} F_{l}^{(k)}}{2^{2 \mu} \Gamma^{2}(\mu+1)} \frac{\bar{Q}_{z-1 / 2}^{\mu}\left(u_{0}\right)}{\bar{P}_{z-1 / 2}^{-\mu}\left(u_{0}\right)},
\end{aligned}
$$

where

$$
\begin{aligned}
& F_{0}^{(0)}=2\left(\xi-\frac{1}{4}\right)\left[z^{2}-\frac{(D-1)^{2}}{4}\right]+z^{2}-z_{m}^{2}, \\
& F_{0}^{(k)}=\left[2\left(1-\frac{1}{D}\right) \xi-\frac{1}{2}\right]\left[z^{2}-\frac{(D-1)^{2}}{4}\right]-(D-1) \xi,
\end{aligned}
$$

and

$F_{1}^{(0)}=2 D(\xi-1 / 4)$,

$F_{1}^{(k)}=2(D-1) \xi-D / 2+1$.

In Eqs. (4.10) and (4.11), $k=1,2, \ldots, D$ and the stresses are isotropic at the center. For $D=3$, Eq. (4.9) is further simplified by using Eq. (3.8). The functions $P_{z-1 / 2}^{-3 / 2}(u)$ and $Q_{z-1 / 2}^{3 / 2}(u)$ in the $l=1$ term of Eq. (4.9) are expressed in terms of the hyperbolic functions by using Eq. (3.7) and the recurrence relations for the associated Legendre functions.

For the shell-induced contribution in the vacuum expectation value of the energy-momentum tensor in the exterior region, $r>r_{0}$, we get 


$$
\begin{aligned}
& \left\langle T_{k}^{k}\right\rangle_{\mathrm{s}}=\frac{a^{-1-D}}{\pi S_{D}} \sum_{l=0}^{\infty} D_{l} \mathrm{e}^{-i \mu \pi} \\
& \times \int_{z_{m}}^{\infty} \mathrm{d} z z \frac{\bar{P}_{z-1 / 2}^{-\mu}\left(u_{0}\right)}{\bar{Q}_{z-1 / 2}^{\mu}\left(u_{0}\right)} \frac{F^{(k)}\left[q_{z-1 / 2}^{\mu}(u)\right]}{\sqrt{z^{2}-z_{m}^{2}}} .
\end{aligned}
$$

For points near the sphere the corresponding asymptotic for the energy density and parallel stresses are given by Eq. (4.7) with the replacement $\left(r_{0}-r\right) \rightarrow\left(r-r_{0}\right)$. Hence, for a nonconformally coupled field, near the boundary these components have the same sign in the exterior and interior regions. For the radial stress we have the same relation (4.8) and for a non-conformally coupled field it has opposite signs inside and outside the sphere.

Now we turn to the asymptotic behavior of the vacuum energy-momentum tensor at large distances from the sphere. In this limit we use Eq. (3.13). The dominant contribution to the integral in Eq. (4.12) comes form the region near the lower limit. For $z_{m}>0$, to the leading order we get (no summation over $k$ )

$$
\begin{aligned}
\left\langle T_{k}^{k}\right\rangle_{\mathrm{s}} \approx & \frac{2^{D-3} \sqrt{\pi z_{m} / r} F^{(k)}\left(z_{m}\right)}{S_{D} a^{D+1} \mathrm{e}^{\left(2 z_{m}+D-1\right) r}} \sum_{l=0}^{\infty} D_{l} \mathrm{e}^{i \mu \pi} \\
& \times \frac{\bar{P}_{z_{m}-1 / 2}^{-\mu}\left(u_{0}\right)}{\bar{Q}_{z_{m}-1 / 2}^{\mu}\left(u_{0}\right)} \frac{\Gamma^{2}\left(z_{m}+1 / 2+\mu\right)}{\Gamma^{2}\left(z_{m}+1\right)}(1+O(1 / r)),
\end{aligned}
$$

where we have defined the functions

$$
\begin{aligned}
& F^{(0)}(z)=(4 \xi-1) z[z+(D-1) / 2], \\
& F^{(1)}(z)=-\frac{1}{2}(D-1)\left[(4 \xi-1) z+2 D\left(\xi-\xi_{D}\right)\right],
\end{aligned}
$$

and $F^{(k)}(z)=2 z F^{(1)}(z) /(1-D)$ for $k=2, \ldots, D$. For minimally and conformally coupled fields $F^{(0)}(z)<0$ and from Eq. (4.13) it follows that the sphere-induced contribution to the vacuum energy of these fields is negative for the Dirichlet boundary condition and positive for the Neumann boundary condition.

For $z_{m}=0$, the leading term in the asymptotic expansion at large distances has the form (no summation over $k$ )

$$
\begin{aligned}
\left\langle T_{k}^{k}\right\rangle_{\mathrm{s}} \approx & \frac{2^{D-3} D F_{(\mathrm{e})}^{(k)} \mathrm{e}^{-(D-1) r}}{S_{D} a^{D+1} r^{2-\delta_{1}^{k}}} \sum_{l=0}^{\infty} D_{l} \mathrm{e}^{i \mu \pi} \\
& \times \frac{\bar{Q}_{-1 / 2}^{\mu}\left(u_{0}\right)}{\bar{P}_{-1 / 2}^{-\mu}\left(u_{0}\right)} \Gamma^{2}(1 / 2+\mu)(1+O(1 / r)),
\end{aligned}
$$

with the coefficients

$F_{(\mathrm{e})}^{(0)}=\xi_{D}(4 \xi-1)$,

$F_{(\mathrm{e})}^{(1)}=(1-D)\left(\xi-\xi_{D}\right), F_{(\mathrm{e})}^{(k)}=\xi-\xi_{D}$,

and with $k=2, \ldots, D$ in the last expression. In this case, for non-conformally coupled fields one has $\left|\left\langle T_{1}^{1}\right\rangle_{\mathrm{s}}\right| \gg\left|\left\langle T_{0}^{0}\right\rangle_{\mathrm{s}}\right|$.
Again, at large distances we have an exponential suppression for both massive and massless fields. For minimally and conformally coupled fields the energy density corresponding to Eq. (4.15) is negative for the Dirichlet boundary condition and positive for the Neumann one. Note that for a sphere in Minkowski bulk the Casimir densities for massive fields are suppressed by the factor $\mathrm{e}^{-2 m \rho}$. For non-conformally coupled massless fields, at large distances the contribution of the $l=0$ mode dominates and all the diagonal components of the vacuum energy-momentum tensor in Minkowski bulk are of the same order. In this case the vacuum expectation values decay as $1 / \rho^{2 D-1}$ for $D \geqslant 3$ and as $1 /\left(\rho^{3} \ln \rho\right)$ for $D=2$ (see Ref. [59]).

In Fig. 2, for a minimally coupled massless field, we have plotted the sphere-induced contribution in the vacuum energy density inside (left panel) and outside (right panel) the $D=3$ spherical shell versus the radial coordinate $r$ for the values of the radius $r_{0}=1,1.5,2$ (numbers near the curves). The full/dashed curves correspond to Dirichlet/Neumann boundary conditions.

In Fig. 3, the sphere-induced contribution in the vacuum energy is displayed as a function of the parameter $\beta$ under the Robin boundary condition for fixed value of $r$ (numbers near the curves) and for $D=3$ sphere with the radius $r_{0}=$ 2. Again, the graphs are plotted for a minimally coupled massless field.

Here we have considered the vacuum expectation value of the bulk energy-momentum tensor. On manifolds with boundaries and for a scalar field with the Robin boundary condition there is also a surface energy-momentum tensor located on the boundary. In the general case of bulk and boundary geometries the expression for the latter is given in Ref. [60] (see also the discussion in Ref. [63-66]). The expectation value of the surface energy-momentum tensor can be evaluated by making use of the corresponding mode-sum with the eigenfunctions given above. However, in this case the renormalization procedure is not reduced to the one in the boundary-free space and additional subtractions are necessary. This procedure can be realized, for example, by using the generalized zeta function technique and will be discussed elsewhere with the total Casimir energy.

For a conformally coupled massless scalar field, the generalization of the results given above for the FriedmannRobertson-Walker backgrounds, described by Eq. (2.2) with a time-dependent scale factor $a=a(t)$, is straightforward. For that, in the expressions for the Wightman function and for the vacuum expectation values of the field squared and the energy-momentum tensor, one should make the replacement $a \rightarrow a(t)$. For non-conformally coupled fields the problem is more complicated. In particular, similar to the case of de Sitter background, we expect that in addition to the diagonal components, the vacuum energy-momentum tensor will 


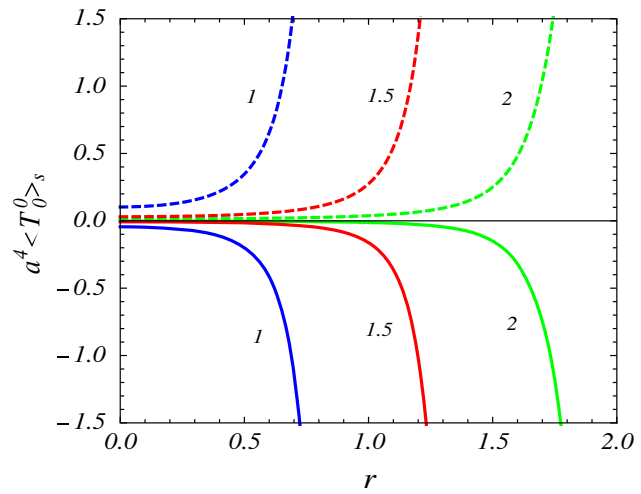

Fig. 2 The sphere-induced vacuum energy density for a minimally coupled $D=3$ massless scalar field as a function of the radial coordinate. The graphs are plotted for several values of the sphere radius

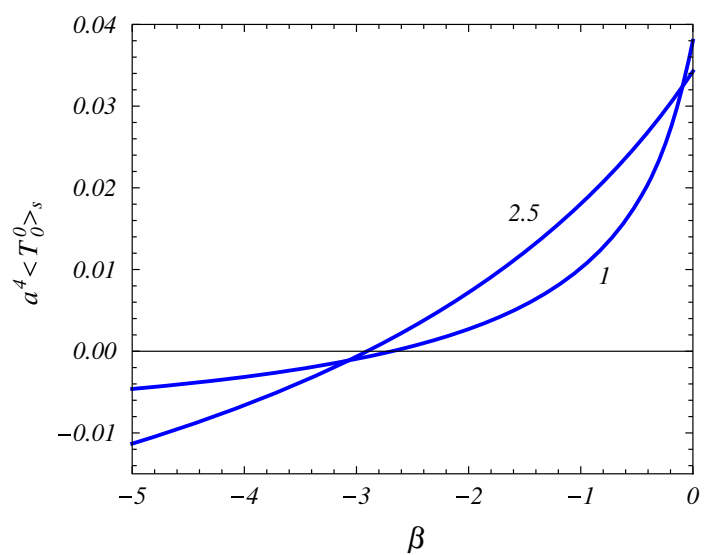

Fig. 3 The sphere-induced vacuum energy density versus the coefficient in the Robin boundary condition in the case of a minimally coupled $D=3$ massless field. For the sphere radius one has $r_{0}=2$ and the numbers near the curves correspond to the values of the radial coordinate

have an off-diagonal component describing the energy flux along the radial direction.

\section{Casimir densities induced by spherical bubbles}

In this section we consider the background spacetime with the geometry described by distinct metric tensors inside and outside a spherical boundary. The interior and exterior gravitational backgrounds may correspond to different vacuum states of a physical system. In this case the sphere serves as a thin-wall approximation of a domain wall interpolating between two coexisting vacua [61]. In what follows we shall refer to the interior region as a bubble.

\subsection{Bubble in a constant curvature space}

First we consider the geometry described by the line element (2.2) in the region $r>r_{0}$ and by

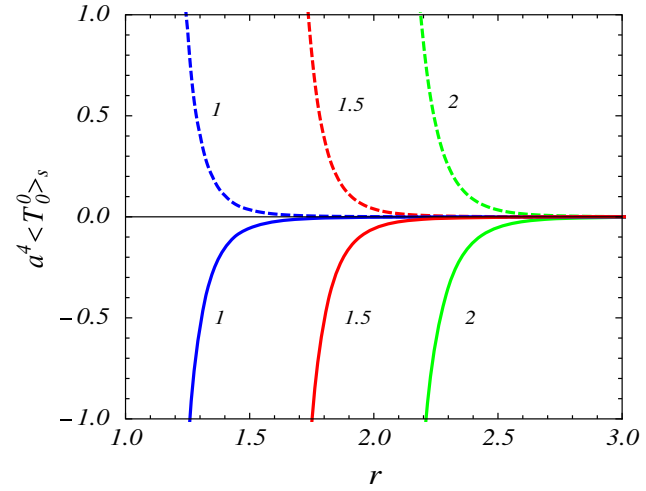

( $r_{0}=1,1.5,2$, numbers near the curves). The left/right panel corresponds to the interior/exterior regions and the full and dashed curves are for the Dirichlet and the Neumann boundary conditions, respectively

$\mathrm{d} s^{2}=\mathrm{e}^{2 u(r)} \mathrm{d} t^{2}-a^{2}\left[\mathrm{e}^{2 v(r)} \mathrm{d} r^{2}+\mathrm{e}^{2 w(r)} \mathrm{d} \Omega_{D-1}^{2}\right]$,

in the region $r<r_{0}$, assuming that the corresponding metric tensor is regular. For generality, the presence of the surface energy-momentum tensor with nonzero components $\tau_{0}^{0}$ and $\tau_{2}^{2}=\cdots=\tau_{D}^{D}$, located at $r=r_{0}$, will be assumed. The continuity of the metric tensor at the separating boundary gives

$u\left(r_{0}\right)=0, v\left(r_{0}\right)=0, w\left(r_{0}\right)=\ln \left(\sinh r_{0}\right)$.

From the Israel matching conditions on the sphere $r=r_{0}$ we get (no summation over $k=2,3, \ldots, D$ )

$8 \pi G a \tau_{0}^{0}=(D-1)\left[w^{\prime}\left(r_{0}\right)-\operatorname{coth} r_{0}\right]$,

$8 \pi G a \tau_{k}^{k}=u^{\prime}\left(r_{0}\right)+(D-2)\left[w^{\prime}\left(r_{0}\right)-\operatorname{coth} r_{0}\right]$,

where $G$ is the gravitational constant. For the trace of the surface energy-momentum tensor one has

$\frac{8 \pi G}{D-1} a \tau=u^{\prime}\left(r_{0}\right)+(D-1)\left[w^{\prime}\left(r_{0}\right)-\operatorname{coth} r_{0}\right]$.

A general problem for the Casimir densities in spherically symmetric spaces with bubbles is considered in Ref. [62]. In that paper the exterior metric was assumed to be asymptotically flat. The latter is not the case for the problem under consideration. However, the main steps for the evaluation are similar and we omit the details.

The mode functions for a scalar field in the interior and exterior regions are presented in the form (2.5). We denote the regular solution of the equation for the radial function in the interior region by $R_{(i) l}(r, E)$ taking it to be real. By taking into account that the energy appears in the equation for the radial function in the form $E^{2}$, we shall also assume that $R_{(i) l}(r,-E)=$ const $\cdot R_{(i) l}(r, E)$. For the radial function in 
the exterior region one has

$$
R_{l}(r)=A_{1} q_{i z-1 / 2}^{-\mu}(u)+A_{2} q_{-i \lambda-1 / 2}^{-\mu}(u) .
$$

The radial functions are continuous at $r=r_{0}$ and for their derivatives one has the jump condition [62]

$R_{l}^{\prime}\left(r_{0}+0\right)-R_{l}^{\prime}\left(r_{0}-0\right)=\frac{16 \pi G \xi}{D-1} a \tau R\left(r_{0}\right)$

This jump comes from the delta function term in the field equation (2.1) contained in the Ricci scalar. From these matching conditions, for the radial functions in the geometry at hand one gets

$R_{l}(r)=C_{\alpha} V_{i z}^{-\mu}(u), r>r_{0}$,

where

$V_{i z}^{-\mu}(u)=\hat{q}_{-i z-1 / 2}^{-\mu}\left(u_{0}\right) q_{i z-1 / 2}^{-\mu}(u)-\hat{q}_{i z-1 / 2}^{-\mu}\left(u_{0}\right) q_{-i z-1 / 2}^{-\mu}(u)$

and

$C_{\alpha}=\frac{R_{(i) l}\left(r_{0}, E\right)}{W_{l}^{(12)}\left(r_{0}\right)}$.

In Eq. (5.8), the notation with hat is defined as

$\hat{F}(u)=\sqrt{u^{2}-1} F^{\prime}(u)-\left[\frac{R_{(i) l}^{\prime}\left(r_{0}, E\right)}{R_{(i) l}\left(r_{0}, E\right)}+\frac{16 \pi G \xi}{D-1} a \tau\right] F(u)$,

where $R_{(i) l}^{\prime}\left(r_{0}, E\right)=\left.\partial_{r} R_{(i) l}(r, E)\right|_{r=r_{0}}$, and

$W_{l}^{(12)}(r)=\sqrt{u^{2}-1} W\left\{q_{i z-1 / 2}^{-\mu}(u), q_{-i z-1 / 2}^{-\mu}(u)\right\}$,

with $W\{f(u), g(u)\}$ being the Wronskian for the enclosed functions. In the normalization condition, similar to Eq. (2.13), the integration goes over both the interior and the exterior regions. However, the dominant contribution comes from large values of $r$. By using the asymptotic for the associated Legendre function, similar to the case of the region outside a spherical boundary with the Robin boundary condition, one gets

$C_{\alpha}^{2}=\frac{\left|\hat{q}_{i z-1 / 2}^{-\mu}\left(u_{0}\right)\right|^{-2}|\Gamma(1-i z)|^{2}}{2 \pi^{2} a^{D} N\left(m_{k}\right) E(z)|\Gamma(1 / 2-i z-\mu)|^{2}}$.

Now substituting the exterior mode functions with the normalization coefficient (5.12) into the mode-sum for the
Wightman function, in the exterior region we get the formula

$$
\begin{aligned}
& W\left(x, x^{\prime}\right)=\sum_{l=0}^{\infty} \frac{(2 l+n) C_{l}^{n / 2}(\cos \theta)}{2 \pi n S_{D} a^{D}} \int_{0}^{\infty} \mathrm{d} z z \sinh (\pi z) \\
& \times|\Gamma(i z+\mu+1 / 2)|^{2} \frac{y_{i z-1 / 2}^{-\mu}(u)\left[y_{i z-1 / 2}^{-\mu}\left(u^{\prime}\right)\right]^{*}}{\hat{q}_{i z-1 / 2}^{-\mu}\left(u_{0}\right)\left[\hat{q}_{i z-1 / 2}^{-\mu}\left(u_{0}\right)\right]^{*}} \frac{\mathrm{e}^{-i E(z) \Delta t}}{E(z)},
\end{aligned}
$$

with the function

$$
\begin{aligned}
y_{i z-1 / 2}^{-\mu}(u)= & \hat{q}_{i z-1 / 2}^{-\mu}\left(u_{0}\right) p_{i z-1 / 2}^{-\mu}(u) \\
& \left.-\hat{p}_{i z-1 / 2}^{-\mu}(u)\right) q_{i z-1 / 2}^{-\mu}(u) .
\end{aligned}
$$

In deriving Eq. (5.13) we have used the relation

$V_{i z}^{-\mu}(u)=-i \pi \frac{\mathrm{e}^{-i \mu \pi} \sinh (\pi z)}{\cos [\pi(i z+\mu)]} y_{i z-1 / 2}^{-\mu}(u)$.

Now by comparing Eq. (5.13) with the expression (2.44) for the corresponding function outside the Robin sphere, we see that Eq. (5.13) differs from Eq. (2.44) by the replacement

$\beta \rightarrow-\frac{R_{(i) l}^{\prime}\left(r_{0}, E\right)}{R_{(i) l}\left(r_{0}, E\right)}-\frac{16 \pi G \xi}{D-1} a \tau$

The further evaluation of the Wightman function is similar to that we have given in Sect. 2 for the exterior region. The only difference is that, after using the identity (2.46) in the integral representation (2.44), in the rotation of the contours of the integrations we should take into account that now the effective Robin coefficient depends on $z$ through $E=E(z)$ (see Eq. (5.16)). Because of our choice of the interior radial function, the logarithmic derivative in Eq. (5.16) is an even function of the energy and the dependence on $z$ gives no additional difficulties in the evaluation process. As a result, the bubble-induced part in the Wightman function is given by Eq. (2.47) where now, in the notation (2.23), we should make the replacement

$\beta \rightarrow-\frac{R_{(i) l}^{\prime}\left(r_{0}, \mathrm{e}^{\pi i / 2} \sqrt{z^{2}-z_{m}^{2}}\right)}{R_{(i) l}\left(r_{0}, \mathrm{e}^{\pi i / 2} \sqrt{z^{2}-z_{m}^{2}}\right)}-\frac{16 \pi G \xi}{D-1} a \tau$,

where the second term in the right-hand side is given by Eq. (5.4). With the same replacement in Eqs. (3.12) and (4.12), we obtain the vacuum expectation values of the field squared and the energy-momentum tensor.

Similar to the case of the Robin sphere, in the geometry of the bubble under consideration, the expectation values of the field squared and the energy-momentum tensor diverge on the separating boundary. It can be seen that (see also Ref. [62]), because of the dependence of the effective Robin coefficient in Eq. (5.17) on the integration variable $z$, the leading terms in the asymptotic expansion of the vacuum expectation 
values over the distance from the boundary vanish and the divergences are weaker compared to the case of the Robin sphere. In particular, the expectation value of the field squared diverges as $\left(r-r_{0}\right)^{2-D}$ and the energy density behaves as $\left(r-r_{0}\right)^{-D}$.

As a simple application of the general results, consider a bubble with the Minkowskian interior with the functions $v(r)=0$ and $w(r)=\ln r$ in Eq. (5.1). For the surface energy density one finds

$\tau_{0}^{0}=\frac{D-1}{8 \pi G a}\left(1 / r_{0}-\operatorname{coth} r_{0}\right)$,

and for the stresses we get (no summation over $k=2, \ldots, D$ ) $\tau_{k}^{k}=(D-2) \tau_{0}^{0} /(D-1)$. Note that $-1<1 / r_{0}-\operatorname{coth} r_{0} \leqslant 0$ for $\infty>r_{0} \geqslant 0$ and the corresponding energy density is always negative. In this special case for the regular radial function in the interior region one has

$R_{(i) l}(r, E)=\frac{\text { const }}{r^{n / 2}} J_{l+n / 2}\left(a r \sqrt{E^{2}-m^{2}}\right)$,

with $J_{v}(x)$ being the Bessel function. Hence, the Casimir densities in the region $r>r_{0}$, induced by the interior Minkowskian geometry are given by Eqs. (3.12) and (4.12), making the replacement

$\beta \rightarrow-\frac{1}{r_{0}}\left[y \frac{I_{l+n / 2}^{\prime}(y)}{I_{l+n / 2}(y)}-\frac{n}{2}+2 \xi(D-1)\left(1-r_{0} \operatorname{coth} r_{0}\right)\right]$

with the notation

$y=r_{0} \sqrt{z^{2}-z_{m}^{2}+m^{2} a^{2}}$,

and with the modified Bessel function $I_{v}(x)$. For the example of a minimally coupled massless scalar field we have checked that there are no bound states in the geometry under consideration.

\subsection{Bubble of a constant curvature in Minkowski spacetime}

Now we consider a background geometry with the line element (2.2) in the region $r<r_{0}$ and with the Minkowskian spacetime in the region $r>r_{0}$. The components of the surface energy-momentum tensor are given by Eq. (5.18) with the opposite signs. In particular, the corresponding energy density is always positive. The Casimir densities in the exterior region are obtained from the general results of Ref. [62] for the special interior geometry under consideration. For the Wightman function one has the decomposition

$W\left(x, x^{\prime}\right)=W_{M}\left(x, x^{\prime}\right)+W_{\mathrm{b}}\left(x, x^{\prime}\right)$, where $W_{M}\left(x, x^{\prime}\right)$ is the Wightman function in the Minkowski spacetime and the part

$$
\begin{aligned}
W_{\mathrm{b}}\left(x, x^{\prime}\right)= & -\sum_{l=0}^{\infty} \frac{(2 l+n) C_{l}^{n / 2}(\cos \theta)}{\pi n S_{D} a^{D-1}\left(r r^{\prime}\right)^{n / 2}} \int_{m a}^{\infty} \mathrm{d} z z \frac{\tilde{I}_{l+n / 2}\left(z r_{0}, z\right)}{\tilde{K}_{l+n / 2}\left(z r_{0}, z\right)} \\
& \times \frac{\cosh \left((\Delta t / a) \sqrt{z^{2}-m^{2} a^{2}}\right)}{\sqrt{z^{2}-m^{2} a^{2}}} K_{l+n / 2}(z r) K_{l+n / 2}\left(z r^{\prime}\right),
\end{aligned}
$$

is induced by the constant curvature bubble. In Eq. (5.23), we have defined the notation

$$
\begin{aligned}
& \tilde{F}(y, z)=y \partial_{y} F(y)-\left[r_{0} \sinh r_{0} \frac{p_{w(z)-1 / 2}^{-\mu \prime}\left(u_{0}\right)}{p_{w(z)-1 / 2}^{-\mu}\left(u_{0}\right)}\right. \\
& \left.\quad+2 \xi(D-1)\left(r_{0} \operatorname{coth} r_{0}-1\right)+\frac{n}{2}\right] F(y),
\end{aligned}
$$

with

$w(z)=\sqrt{z^{2}-m^{2} a^{2}+z_{m}^{2}}$.

Note that under the condition $z_{m}^{2} \geqslant 0$ the function $w(z)$ is real and the Minkowskian vacuum in the exterior region is stable. The corresponding Casimir densities in the exterior region are obtained from the expressions in Ref. [62] by the replacements $\tilde{F}(y) \rightarrow \tilde{F}(y, z)$ with Eq. (5.24) and $F=$ $I_{l+n / 2}, K_{l+n / 2}$.

\section{Conclusion}

We have investigated the properties of the scalar vacuum in a constant negative curvature space induced by a spherical boundary on which the field operator obeys the Robin boundary condition. General values of the spatial dimension and of the curvature coupling parameter are considered. For the coefficient in the Robin boundary condition there is a critical value, above which the scalar vacuum becomes unstable. The properties of the quantum vacuum are encoded in twopoint functions. We have considered the positive-frequency Wightman function and the other function can be evaluated in a similar way. In order to evaluate the Wightman function we employed the direct summation over the complete set of modes. In the region inside the sphere, the eigenmodes of the field are expressed in terms of the zeros of the combination of the associated Legendre function and its derivative with respect to the order (see Eq. (2.22)). In the mode-sum for the Wightman function, for the summation of the series over these zeros we have used the formula (8.4), derived from the generalized Abel-Plana formula. This allowed us to separate the part corresponding to the boundary-free geometry and to present the sphere-induced part in terms of a 
rapidly convergent integral. In this form the explicit knowledge of the eignemodes is not required. In addition, with the decomposition into the boundary-free and boundary-induced contributions, the renormalization of the vacuum expectation values in the coincidence limit is reduced to the one for the boundary-free geometry. We have provided a similar decomposition for the exterior region as well.

As an important local characteristic of the vacuum state, in Sect. 3 we consider the vacuum expectation value of the field squared. The corresponding expressions for the sphereinduced parts in the interior and exterior regions are given by Eqs. (3.2) and (3.12). These parts are negative for the Dirichlet boundary condition and positive for the Neumann boundary condition. The expectation value of the field squared diverges on the boundary. The leading term in the asymptotic expansion over the distance from the sphere is given by Eq. (3.5) and coincides with that for a sphere in Minkowski bulk. In this limit the dominant contribution comes from the wavelengths smaller than the curvature radius of the background and the effects of the gravitational field are small. In the opposite limit of large distances, the effects of gravity are decisive. The asymptotic behavior in this region depends on whether the parameter $z_{m}$, defined by Eq. (2.11), is zero or not. For $z_{m}>0$, the leading term is given by Eq. (3.14) and the boundary-induced vacuum expectation value is exponentially suppressed. For $z_{m}=0$ the leading term is given by Eq. (3.15). The decay in this case is again exponential, though relatively weaker. Consequently, for both massive and massless fields the suppression of the boundary-induced vacuum expectation values at large distances is exponential. This is in contrast to the case of Minkowskian bulk, where the decay for massless fields has a power-law behavior.

Another important characteristic of the vacuum is the vacuum expectation value of the energy-momentum tensor. This expectation value is diagonal and the expressions for the sphere-induced contributions are given by Eqs. (4.3) and (4.12) for the interior and exterior regions, respectively. Near the sphere, the leading term in the energy density and parallel stresses is given by Eq. (4.7). Once again, this term coincides with the corresponding asymptotic for a spherical shell in flat spacetime. For the leading term in the normal stress one has the expression (4.8) and the corresponding divergence is weaker. The asymptotics at large distances are given by Eqs. (4.13) and (4.15) for the cases $z_{m}>0$ and $z_{m}=0$, respectively. In both cases the vacuum expectation values are suppressed by the factor $\mathrm{e}^{-\left(2 z_{m}+D-1\right) r}$. At large distances and for non-conformally coupled fields one has $\left|\left\langle T_{1}^{1}\right\rangle_{\mathrm{s}}\right| \gg\left|\left\langle T_{0}^{0}\right\rangle_{\mathrm{s}}\right|$. Again, in contrast to the Minkowskian case, the expectation values are exponentially suppressed for both massive and massless fields. This feature is observed also in the Casimir problems on the background of the antide Sitter spacetime, which presents another example of a negative curvature space. In a de Sitter spacetime, having a positive curvature, the situation is opposite: at large distances from the boundary the local vacuum expectation values for both massive and massless fields exhibit a power-law behavior.

We have generalized the results for the vacuum expectation values to the backgrounds described by two distinct spherically symmetric metric tensors in the regions separated by a spherical boundary. The geometry of one region affects the properties of the vacuum in the other region leading to the gravitationally induced Casimir effect. We have considered the interior geometry described by the line element (5.1), and the exterior geometry, as before, corresponds to a constant curvature space. In addition, we have assumed the presence of the surface energy-momentum tensor located on the separating boundary. The corresponding jump conditions on the normal derivatives of the metric tensor are obtained from the Israel matching conditions, whereas the boundary conditions on the field operator are obtained from the field equation. In this way, we have shown that the bubble-induced contributions in the Wightman function and in the vacuum expectation values of the field squared and the energy-momentum tensor are obtained from the expressions for the spherical shell with the Robin boundary condition by the replacement (5.17) of the coefficient. As an example of the exterior geometry we have considered the case of the Minkowskian bubble. In this case the replacement needed is given by Eq. (5.20). Note that in the geometry with bubbles the divergences on the boundary are weaker compared with the case of the Robin sphere. We have also considered a bubble with a negative constant curvatures space in the Minkowski bulk. The corresponding expressions for the local characteristics are obtained from the general formulas in Ref. [62] with the notation with tilde defined by Eq. (5.24).

The generalization of the results given above for the Friedmann-Robertson-Walker cosmological models with a time-dependent scale factor is straightforward in the case of a conformally coupled massless field. This requires the replacement $a \rightarrow a(t)$ in the expressions for the Wightman function and for the vacuum expectation values. In the special case $D=2$, the results obtained can be applied to negatively curved graphene structures (for this type of structures, see Ref. [9]), described in the long-wavelength regime by an effective three-dimensional relativistic field theory. The latter, in addition to the well-known Dirac fermions describing the low-energy excitations of the electronic subsystem, involves scalar and gauge fields originating from the elastic properties and describing disorder phenomena, like the distortions of the graphene lattice and structural defects (see, for example, Refs. [68,69] and references therein). In this setup, the spherical boundary models the edge of a negatively curved graphene sheet. The boundary condition we have used ensures the zero flux of a scalar field through the boundary. The Casimir effect in graphene-made structures 
with zero curvature, like cylindrical and toroidal carbon nanotubes, has been recently discussed in Refs. [70-72].

Acknowledgments A. A. S. was supported by the State Committee of Science Ministry of Education and Science RA, within the frame of Grant No. SCS 13-1C040.

Open Access This article is distributed under the terms of the Creative Commons Attribution License which permits any use, distribution, and reproduction in any medium, provided the original author(s) and the source are credited.

Funded by $\mathrm{SCOAP}^{3}$ / License Version CC BY 4.0.

\section{Appendix A: On the eigenvalues inside a sphere}

As has been shown in Sect. 2, the eigenvalues of the quantum number $z$ in the region inside a spherical shell with the boundary condition (2.3) are zeros of the function $\bar{P}_{i z-1 / 2}^{-\mu}(u)$ for a given value of $u>1$. The corresponding positive roots we have denoted by $z_{k}, k=1,2, \ldots$ From the relation $\bar{P}_{i z-1 / 2}^{-\mu}(u)=\bar{P}_{-i z-1 / 2}^{-\mu}(u)$ it follows that $z=-z_{k}$ are zeros of the function $\bar{P}_{i z-1 / 2}^{-\mu}(u)$ as well. Note that the latter can also be written in the form

$\bar{P}_{i z-1 / 2}^{-\mu}(u)=\left(u^{2}-1\right)^{D / 4} \tilde{p}_{i z-1 / 2}^{-\mu}(u)$,

where, for a given function $F(u)$, we have defined the notation

$\tilde{F}(u)=A F(u)-\delta_{(j)} \frac{B}{a} \sqrt{u^{2}-1} \partial_{u} F(u)$,

and for the interior region $\delta_{(j)}=1$. From (7.1) we see that for $u>1$ the points $z=z_{k}$ are the zeros of the function $\tilde{p}_{i z-1 / 2}^{-\mu}(u)$. First of all let us show that these zeros are simple.

By taking into account that the function $R_{l}(r)=$ $p_{i z-1 / 2}^{-\mu}(\cosh r)$ is a solution of Eq. (2.9), the following integration formula can be obtained:

$$
\begin{aligned}
& \int_{1}^{u} \mathrm{~d} x\left(x^{2}-1\right)^{D / 2-1}\left[p_{i z-1 / 2}^{-\mu}(x)\right]^{2}=\frac{\left(u^{2}-1\right)^{D / 2}}{2 z} \\
& \quad \times\left\{\left[\partial_{z} p_{i z-1 / 2}^{-\mu}(u)\right] \partial_{u} p_{i z-1 / 2}^{-\mu}(u)\right. \\
& \left.\quad-p_{i z-1 / 2}^{-\mu}(u) \partial_{z} \partial_{u} p_{i z-1 / 2}^{-\mu}(u)\right\} .
\end{aligned}
$$

From this, for $z=z_{k}$ one gets

$$
\begin{aligned}
& \int_{1}^{u} \mathrm{~d} x\left(x^{2}-1\right)^{D / 2-1}\left[p_{i z-1 / 2}^{-\mu}(x)\right]^{2} \\
& \quad=\left.\frac{\left(u^{2}-1\right)^{(D-1) / 2}}{2 z B_{1} / a} p_{i z-1 / 2}^{-\mu}(u) \partial_{z} \tilde{p}_{i z-1 / 2}^{-\mu}(u)\right|_{z=z_{k}} .
\end{aligned}
$$

The left-hand side of this relation is positive (recall that $p_{i z-1 / 2}^{-\mu}(x)$ is a real function) and, hence, $\left.\partial_{z} \tilde{p}_{i z-1 / 2}^{-\mu}(u)\right|_{z=z_{k}}$ $\neq 0$. This shows that the zeros $z_{k}$ are simple.
The asymptotic expression for large positive zeros is found by using the asymptotic formula

$$
\begin{aligned}
& P_{i z-1 / 2}^{-\mu}(u) \sim \sqrt{\frac{2}{\pi}} \frac{z^{-\mu-1 / 2}}{\sqrt{u^{2}-1}} \\
& \quad \times \sin (z \operatorname{arccosh} u-\pi \mu / 2+\pi / 4)\left(1+O\left(1 / z^{2}\right)\right),
\end{aligned}
$$

for $z \gg 1$. From here we see that

$z_{k} \approx \pi \frac{2 k+\mu+1 / 2-\delta_{0 B}}{2 \operatorname{arccosh} u}+O(1 / k)$.

Now let us discuss possible purely imaginary zeros. With fixed $u$ and $l$, the function $\bar{P}_{i z-1 / 2}^{-\mu}(u)$ has no purely imaginary zeros for sufficiently small values of the ratio $\beta=A a / B$. With increasing $\beta$, started from some critical value $\beta_{l}^{(1)}(u)$, a pair of purely imaginary zeros $z= \pm i \eta, \eta>0$, appears. The critical value $\beta_{l}^{(1)}(u)$ increases with increasing $l$ and, hence, the purely imaginary zero first appears for the mode $l=0$ (see the left panel of Fig. 1, where in the case $D=3$ the critical value is plotted versus $r_{0}=\operatorname{arccosh} u$ for different values of $l$ ). By taking into account that for $r \gg 1$ one has

$P_{z-1 / 2}^{-\mu}(\cosh r) \approx \frac{\Gamma(z) \mathrm{e}^{(z-1 / 2) r}}{\sqrt{\pi} \Gamma(1 / 2+z+\mu)}$,

it is seen that for large values of $u$ we have $\eta \approx \beta+(D-$ $1) / 2$ and $\beta_{l}^{(1)}(u) \rightarrow-(D-1) / 2$ for $u \rightarrow \infty$. There are no purely imaginary zeros for $\beta<-(D-1) / 2$. With the further increase of $\beta>\beta_{l}^{(1)}(u)$ the value of $\eta$ increases and for the second critical value $\beta=\beta_{l}^{(2)}(u)$ the energy of the corresponding mode becomes zero. This corresponds to $\eta=z_{m}$. For $\beta>\beta_{l}^{(2)}(u)$ the energy of the mode becomes imaginary, which signals the instability of the vacuum.

\section{Appendix B: Summation formula}

In this appendix, by making use of the generalized AbelPlana formula $[73,74]$ (see also, [75]), we derive a summation formula for the series over the zeros of the function

$\bar{P}_{i z-1 / 2}^{-\mu}(u)=A(u) P_{i z-1 / 2}^{-\mu}(u)+B(u) \partial_{u} P_{i z-1 / 2}^{-\mu}(u)$,

with respect to $z$, for given $u>1$ and $\mu \geqslant 0$. In general, the functions $A(u)$ and $B(u)$ can be different from those in Eq. (2.24). We shall denote the positive zeros, arranged in ascending order, by $z_{k}, k=1,2, \ldots$, assuming that they are simple. The summation formula can be obtained in a way similar to that used in Ref. [58] for the special case $A(u)=1$ and $B(u)=0$ and we shall outline the main steps only (for the summation formula over the zeros of the combination 
of the associated Legendre functions of the first and second kinds see Ref. [67]).

We substitute in the generalized Abel-Plana formula

$$
\begin{aligned}
& f(z)=\sinh (\pi z) h(z) \\
& g(z)=\frac{\mathrm{e}^{i \mu \pi} h(z)}{\pi i \bar{P}_{i z-1 / 2}^{-\mu}(u)} \sum_{j= \pm} \cos [\pi(\mu-j i z)] \bar{Q}_{j i z-1 / 2}^{-\mu}(u)
\end{aligned}
$$

where the function $h(z)$ is analytic in the right-half plane of the complex variable $z=x+i y$. The function $g(z)$ has simple poles at $z=z_{k}$. For functions obeying the condition

$|h(z)|<\varepsilon(x) \exp (c y \operatorname{arccosh} u),|z| \rightarrow \infty$,

with $c<2, \varepsilon(x) \mathrm{e}^{\pi x} \rightarrow 0$ for $x \rightarrow+\infty$, the following formula is obtained:

$$
\begin{aligned}
& \sum_{k=1}^{\infty} T_{\mu}\left(z_{k}, u\right) h\left(z_{k}\right)=\frac{\mathrm{e}^{-i \mu \pi}}{2} \int_{0}^{\infty} \mathrm{d} x \sinh (\pi x) h(x) \\
& \quad-\frac{1}{2 \pi} \int_{0}^{\infty} \mathrm{d} x \frac{\bar{Q}_{x-1 / 2}^{-\mu}(u)}{\bar{P}_{x-1 / 2}^{-\mu}(u)} \cos [\pi(\mu+x)] \sum_{j= \pm} h\left(x \mathrm{e}^{j \pi i / 2}\right) .
\end{aligned}
$$

Here we have introduced the notation

$T_{\mu}(z, u)=\frac{\bar{Q}_{i z-1 / 2}^{-\mu}(u)}{\partial_{z} \bar{P}_{i z-1 / 2}^{-\mu}(u)} \cos [\pi(\mu-i z)]$.

Equation (8.4) is also valid for some functions having branch points on the imaginary axis, for example, in the case of functions of the form $h(z)=F(z) /\left(z^{2}+z_{0}^{2}\right)^{1 / 2}$, with $F(z)$ being an analytic function. Note that, in the physical problem, we have considered the branch points correspond to $z=$ $\pm i z_{m}$. Adding the corresponding residue terms in the righthand side, Eq. (8.4) can be generalized for functions $h(z)$ having poles in the right-half plane.

An equivalent expression for $T_{\mu}(z, u)$ is obtained by using the relation

$\bar{Q}_{i z-1 / 2}^{-\mu}(u)=\frac{B(u) \mathrm{e}^{-i \mu \pi} \Gamma(i z-\mu+1 / 2)}{\Gamma(i z+\mu+1 / 2)\left(1-u^{2}\right) P_{i z-1 / 2}^{-\mu}(u)}$,

with $z=z_{k}$. This formula follows from the Wronskian relation for the associated Legendre functions. Now, from Eq. (B.5) for $z=z_{k}$ one gets

$T_{\mu}(z, u)=\frac{\pi \mathrm{e}^{-i \mu \pi} B(u)|\Gamma(\mu+i z+1 / 2)|^{-2}}{\left(1-u^{2}\right) P_{i z-1 / 2}^{-\mu}(u) \partial_{z} \bar{P}_{i z-1 / 2}^{-\mu}(u)}$.

Equation (8.4) can be generalized for the case when the function $\bar{P}_{i z-1 / 2}^{-\mu}(u)$ has purely imaginary zeros at the points $z= \pm i y_{k}, y_{k}>0, k=1,2, \ldots$, under the assumption that the function $h(z)$ obeys the condition

$h(z)=-h\left(z \mathrm{e}^{-\pi i}\right)+o\left(z-i y_{k}\right), z \rightarrow i y_{k}$.

Assuming that the zeros are simple, in this case, on the righthand side of Eq. (8.4) we have to add the term

$-\left.i \sum_{k} \frac{\bar{Q}_{y-1 / 2}^{-\mu}(u)}{\partial_{y} \bar{P}_{y-1 / 2}^{-\mu}(u)} \cos [\pi(\mu-y)] h(i y)\right|_{y=y_{k}}$,

at these poles and take the principal value of the second integral on the right-hand side. The latter exists due to the condition (8.8). By using the relation (8.6) the term (8.9) can also be written in the form

$$
\begin{aligned}
& i \sum_{k} \frac{\pi B(u) \mathrm{e}^{-i \mu \pi}}{P_{y-1 / 2}^{-\mu}(u) \partial_{y} \bar{P}_{y-1 / 2}^{-\mu}(u)} \\
& \quad \times\left.\frac{\left(u^{2}-1\right)^{-1} h(i y)}{\Gamma(\mu+y+1 / 2) \Gamma(\mu-y+1 / 2)}\right|_{y=y_{k}} .
\end{aligned}
$$

A physical example with purely imaginary modes using this result is discussed in Sect. 2.

\section{References}

1. N.D. Birrell, P.C.W. Davies, Quantum Fields in Curved Space (Cambridge University Press, Cambridge, 1982)

2. A.A. Grib, S.G. Mamayev, V.M. Mostepanenko, Vacuum Quantum Effects in Strong Fields (Friedmann Laboratory Publishing, St. Petersburg, 1994)

3. V. Mukhanov, S. Winitzki, Introduction to Quantum Effects in Gravity (Cambridge University Press, Cambridge, 2007)

4. L. Parker, D. Toms, Quantum Field Theory in Curved Spacetime: Quantized Fields and Gravity (Cambridge University Press, Cambridge, 2009)

5. S. Hollands, R.M. Wald, arXiv:1401.2026

6. A.D. Linde, Particle Physics and Inflationary Cosmology (Harwood Academic Publishers, Chur, 1990)

7. B.A. Bassett, S. Tsujikawa, D. Wands, Rev. Mod. Phys. 78, 537 (2008)

8. A. Cortijo, F. Guinea, M.A.H. Vozmediano, J. Phys. A Math. Theor. 45, 383001 (2012)

9. A. Iorio, G. Lambiase, arXiv:1308.0265

10. F.J. Tipler, Phys. Rep. 137, 231 (1986)

11. G. Esposito, A. Yu. Kamenshchik, G. Pollifrone, Euclidean Quantum Gravity on Manifolds with Boundary (Kluwer, Dordrecht, 1997)

12. V.M. Mostepanenko, N.N. Trunov, The Casimir Effect and its Applications (Clarendon, Oxford, 1997)

13. E. Elizalde, S.D. Odintsov, A. Romeo, A.A. Bytsenko, S. Zerbini, Zeta Regularization Techniques with Applications (World Scientific, Singapore, 1994)

14. K.A. Milton, The Casimir Effect: Physical Manifestation of ZeroPoint Energy (World Scientific, Singapore, 2002)

15. M. Bordag, G.L. Klimchitskaya, U. Mohideen, V.M. Mostepanenko, Advances in the Casimir Effect (Oxford University Press, Oxford, 2009) 
16. Casimir Physics. ed. by D. Dalvit, P. Milonni, D. Roberts, F. da Rosa, Lecture Notes in Physics, vol. 834 (Springer, Berlin, 2011)

17. W. Goldberger, I. Rothstein, Phys. Lett. B 491, 339 (2000)

18. S. Nojiri, S.D. Odintsov, S. Zerbini, Class. Quantum Grav. 17, 4855 (2000)

19. A. Flachi, D.J. Toms, Nucl. Phys. B 610, 144 (2001)

20. J. Garriga, O. Pujolàs, T. Tanaka, Nucl. Phys. B 605, 192 (2001)

21. E. Elizalde, S. Nojiri, S.D. Odintsov, S. Ogushi, Phys. Rev. D 67, 063515 (2003)

22. A. Knapman, D.J. Toms, Phys. Rev. D 69, 044023 (2004)

23. A.A. Saharian, Nucl. Phys. B 712, 196 (2005)

24. A.A. Saharian, Phys. Rev. D 70, 064026 (2004)

25. A. Flachi, A. Knapman, W. Naylor, M. Sasaki, Phys. Rev. D 70, 124011 (2004)

26. E. Elizalde, S. Nojiri, S.D. Odintsov, P. Wang, Phys. Rev. D 71, 103504 (2005)

27. A.A. Saharian, A.L. Mkhitaryan, J. High Energy Phys. 08, 063 (2007)

28. M. Frank, I. Turan, L. Ziegler, Phys. Rev. D 76, 015008 (2007)

29. E. Elizalde, S.D. Odintsov, A.A. Saharian, Phys. Rev. D 79, 065023 (2009)

30. L.P. Teo, Phys. Lett. B 682, 259 (2009)

31. E. Elizalde, S.D. Odintsov, A.A. Saharian, Phys. Rev. D 87, 084003 (2013)

32. A. Flachi, J. Garriga, O. Pujolàs, T. Tanaka, J. High Energy Phys. 08, 053 (2003)

33. A. Flachi, O. Pujolàs, Phys. Rev. D 68, 025023 (2003)

34. A.A. Saharian, Phys. Rev. D 73, 044012 (2006)

35. A.A. Saharian, Phys. Rev. D 73, 064019 (2006)

36. A.A. Saharian, Phys. Rev. D 74, 124009 (2006)

37. E. Elizalde, M. Minamitsuji, W. Naylor, Phys. Rev. D 75, 064032 (2007)

38. R. Linares, H.A. Morales-Técotl, O. Pedraza, Phys. Rev. D 77, $066012(2008)$

39. M. Frank, N. Saad, I. Turan, Phys. Rev. D 78, 055014 (2008)

40. M.R. Setare, R. Mansouri, Class. Quantum Grav. 18, 2659 (2001)

41. A.A. Saharian, M.R. Setare, Phys. Lett. B 584, 306 (2004)

42. M.R. Setare, Phys. Lett. B 637, 1 (2006)

43. A.A. Saharian, T.A. Vardanyan, Class. Quantum Grav. 26, 195004 (2009)

44. E. Elizalde, A.A. Saharian, T.A. Vardanyan, Phys. Rev. D 81, $124003(2010)$

45. P. Burda, JETP Lett. 93, 632 (2011)
46. A.A. Saharian, Int. J. Mod. Phys. A 26, 3833 (2011)

47. K.A. Milton, A.A. Saharian, Phys. Rev. D 85, 064005 (2012)

48. A.A. Saharian, A.S. Kotanjyan, H.A. Nersisyan, Phys. Lett. B 728, $141(2014)$

49. A.A. Saharian, V.F. Manukyan, arXiv: 1406.0393

50. S. Bellucci, A.A. Saharian, Phys. Rev D 88, 064034 (2013)

51. H.B.G. Casimir, Physica (Amsterdam) 19, 846 (1953)

52. A.S. Wightman, Fortschr. Phys. 44, 143 (1996)

53. R. Camporesi, Phys. Rep. 196, 1 (1990)

54. A.A. Bytsenko, G. Cognola, L. Vanzo, S. Zerbini, Phys. Rep. 266, 1 (1996)

55. Handbook of Mathematical Functions. ed. by M. Abramowitz, I.A. Stegun (Dover, New York, 1972)

56. A. Erdélyi et al., Higher Transcendental Functions, vol. 2 (McGraw Hill, New York, 1953)

57. A.A. Grib, B.A. Levitskii, V.M. Mostepanenko, Theor. Math. Phys. 19, 349 (1974)

58. A.A. Saharian, J. Phys. A Math. Theor. 41, 415203 (2008)

59. A.A. Saharian, Phys. Rev. D 63, 125007 (2001)

60. A.A. Saharian, Phys. Rev. D 69, 085005 (2004)

61. F.R. Klinkhamer, G.E. Volovik, Phys. Lett. A 347, 8 (2005)

62. S. Bellucci, A.A. Saharian, A.H. Yeranyan, Phys. Rev. D 89, 105006 (2014)

63. G. Kennedy, R. Critchley, J.S. Dowker, Ann. Phys. (N.Y.) 125, 346 (1980)

64. S.L. Lebedev, Phys. Atom. Nucl. 64, 1337 (2001)

65. A. Romeo, A.A. Saharian, J. Phys. A 35, 1297 (2002)

66. S.A. Fulling, J. Phys. A 36, 6857 (2003)

67. A.A. Saharian, J. Phys. A Math. Theor. 42, 465210 (2009)

68. R. Jackiw, S.-Y. Pi, Phys. Rev. Lett. 98, 266402 (2007)

69. O. Oliveira, C.E. Cordeiro, A. Delfino, W. de Paula, T. Frederico, Phys. Rev. B 83, 155419 (2011)

70. S. Bellucci, A.A. Saharian, Phys. Rev. D 80, 105003 (2009)

71. E. Elizalde, S.D. Odintsov, A.A. Saharian, Phys. Rev. D 83, 105023 (2011)

72. S. Bellucci, A.A. Saharian, Phys. Rev. D 87, 025005 (2013)

73. A.A. Saharian, Izv. AN Arm. SSR. Matematika 22, 166 (1987)

74. A.A. Saharian, Sov. J. Contemp. Math. Anal. 22, 70 (1987)

75. A.A. Saharian, The generalized Abel-Plana formula with applications to Bessel functions and Casimir effect (Yerevan State University Publishing House, Yerevan, 2008); Report No. ICTP/2007/082. arXiv:0708.1187 